\title{
Self-consistent Green's functions formalism with three-body interactions
}

\author{
Arianna Carbone, ${ }^{1}$ Andrea Cipollone, ${ }^{2}$ Carlo Barbieri, ${ }^{2}$ Arnau Rios,${ }^{2}$ and Artur Polls ${ }^{1}$ \\ ${ }^{1}$ Departament d' Estructura i Constituents de la Matèria and Institut de Ciències del Cosmos, \\ Universitat de Barcelona, E-08028 Barcelona, Spain \\ ${ }^{2}$ Department of Physics, Faculty of Engineering and Physical Sciences, University of Surrey, Guildford, Surrey GU2 7XH, United Kingdom
}

(Received 14 October 2013; published 27 November 2013)

\begin{abstract}
We extend the self-consistent Green's functions formalism to take into account three-body interactions. We analyze the perturbative expansion in terms of Feynman diagrams and define effective one- and two-body interactions, which allows for a substantial reduction of the number of diagrams. The procedure can be taken as a generalization of the normal ordering of the Hamiltonian to fully correlated density matrices. We give examples up to third order in perturbation theory. To define nonperturbative approximations, we extend the equation-of-motion method in the presence of three-body interactions. We propose schemes that can provide nonperturbative resummation of three-body interactions. We also discuss two different extensions of the Koltun sum rule to compute the ground state of a many-body system.
\end{abstract}

DOI: 10.1103/PhysRevC.88.054326 PACS number(s): 21.45.Ff, 21.30.-x, 21.60.De, 24.10.Cn

\section{INTRODUCTION}

The description of quantum many-body systems, whether of nuclear, atomic or molecular nature, is an everlasting challenge of theoretical physics [1,2]. Even if the Hamiltonian is well known, the formalism needed to describe such systems can be baffling. One of the issues is that the actual inter-particle interactions in the medium can be very different from those in free space. For strongly correlated many-body systems, ordinary perturbation theory must be replaced by methods which perform an all-order summation of Feynman diagrams. The self-consistent Green's functions (SCGF) formalism was precisely devised to treat the correlated behavior of such systems [3]. The time-dependent many-body field correlation functions, also called Green's functions (GFs) or propagators, contain information associated with the addition or removal of a given number of particles from the correlated ground state. Consequently, they can be used to obtain invaluable microscopic information on the many-particle system. More importantly, knowledge of the $N$-body GFs translates into the ability of computing all $N$-body operators and hence provides access to a wide range of observables. At the one-body level, the nonperturbative nature of the system is taken into account through the self-consistent solution of the Dyson equation [4].

The original many-body Green's functions formalism dates back to the 1960s [2,5,6]. In the past few decades, computational techniques have gradually improved to the point of allowing for fully ab initio studies that take into account beyond-mean-field correlations. First-principles calculations are now routinely performed in solid state $[7,8]$, atomic and molecular physics [9-12], and nuclear structure [13,14]. In nuclear physics, finite nuclei have been studied using a variety of techniques, including the SCGF approach. The Faddeev random phase approximation (FRPA) was used to describe closed-shell isotopes $[15,16]$. Medium-mass nuclei with open shells have been tackled within the Gorkov-Green's function method [17]. The behavior of correlations in infinite nuclear matter was extensively studied using ladder summations [18-21].
Initially, the many-body Green's functions framework was developed with Hamiltonians containing up to two-body (2B) interactions in mind. In the specific case of nuclear systems, however, three-body (3B) interactions play a substantial role. In infinite matter, three-body forces (3BFs) are long thought to be responsible for saturation [21-27]. Ab initio calculations of light nuclei have pointed towards the essential role played by $3 \mathrm{~B}$ interactions, particularly in reproducing the correct groundand excited-state properties [28-31]. Recent breakthroughs in a wide variety of many-body techniques also indicate that $3 \mathrm{~B}$ interactions play a role in medium-mass nuclei [32-35]. In spite of all these advances, many-body theory with underlying 3B interactions has only been pushed forward in an intermittent fashion [31,36-38].

Our aim here is to develop the formal tools needed to include 3B interactions in nonperturbative calculations within the SCGF formalism. Although our main motivation is nuclear systems, the formalism can be easily applied to other manybody systems. Such an approach is pivotal both to provide theoretical foundations to approximations made so far and to advance the many-body formalisms for much-needed $a b$ initio nuclear structure. In the present paper, we present the extension of the SCGF formalism to include 3BFs by working out in full the first orders of the perturbation expansion and the self-consistent equations of motion. Our approach is mainly practical. We want to put forward the basic rules that are needed to extend present calculations to include 3B interactions. These include extensions in the perturbative expansion, Feynman diagram rules, and the equation-of-motion (EOM) method. Moreover, we extend the Galitskii-Migdal-Koltun (GMK) sum rule to compute the total energy of the system including such interactions $[39,40]$. In principle, the approach is also able to incorporate $3 \mathrm{~B}$ correlations in the many-body wave function, but we will not discuss these explicitly here [41]. We will also not comment on the actual numerical implementation of the approach, which can be found elsewhere in the literature $[27,35]$.

This study is made all the more timely in view of the notable recent efforts in improving the description of the 
strong interaction acting between nucleons. Realistic, phaseshift-equivalent $2 \mathrm{~B}$ potentials have been traditionally used both in finite and infinite matter calculations [42-44]. Using such interactions as a starting point, however, one needs to evaluate the associated 3B forces consistently. Traditionally, this was done either phenomenologically [45] or through an extension of the meson-exchange picture [22]. In the last decade, however, a more systematic approach was devised, based on applying effective field theory to low-energy QCD [46,47]. A particularly appealing advantage of this approach is that it naturally gives rise to $2 \mathrm{~B}, 3 \mathrm{~B}$, and many-body interactions as the order of the expansion increases. This avoids the somewhat ad hoc adjustment of different ingredients in $2 \mathrm{~B}$ and $3 \mathrm{~B}$ potentials.

Another important motivation to develop a formalism that explicitly includes 3BFs comes from recent advances in developing low-momentum interactions [48]. These approaches reduce the computational effort on the many-body side by taming the strong force using renormalization group techniques. However, this results in the appearance of induced 3BFs (and other many-body forces). A number of approximations have been proposed to include both contributions in different many-body calculations [25,27,31-33,35,37]. Most of these approaches involve, at some level, a normal ordering of the Hamiltonian to average on the third, spectator particle. We will show that such an approach is justified within the GFs formalism, provided that a class of interaction-irreducible diagrams are discarded to avoid double countings. Incidentally, the latter approach goes beyond the usual normal ordering of perturbation theory by incorporating fully correlated density matrices in the averaging procedure.

Induced 3BFs are not exclusive to nuclear physics, as they arise from any truncation in the many-body model space. They play a role in a variety of other fields of physics $[49,50]$. The bare and the induced $3 \mathrm{~B}$ interactions, however, are treated on the same footing from a many-body perspective. Hence, techniques that deal with $3 \mathrm{BF}$ and $3 \mathrm{~B}$ correlations within a many-body system are needed to describe such systems. The developments proposed here are of relevance for applications on systems where induced many-body forces play a role.

This paper is organized as follows. In Sec. II we exploit effective one-body (1B) and 2B potentials to group diagrams in the self-energy in a more compact form. We benchmark this approach by explicitly presenting the expansion of the single-particle (SP) GF up to third order in perturbation theory. We study, in Sec. III, the hierarchy of EOMs including $3 \mathrm{BF}$ s by means of the interacting vertex $\Gamma$ functions. There, the truncation of $\Gamma$ is presented up to second order in the perturbative expansion. We also show that this truncation leads to the irreducible self-energy diagrams obtained in Sec. II. Of relevance for practical applications, we present an improved ladder and ring summation that includes 3BFs. Section IV is devoted to the GMK sum rule and its use for the calculation of the ground-state energy of the many-body system. Two extensions of this rule are presented which account for $3 \mathrm{BF}$. Appendix A provides a revision of Feynman diagrams and rules with 3 BFs. We pay particular attention to additional symmetry factors due to equivalent groups of lines. The proof for the expressions of the effective $1 \mathrm{~B}$ and $2 \mathrm{~B}$ interactions at arbitrary orders in perturbation theory is presented in Appendix B.

\section{PERTURBATIVE EXPANSION OF THE ONE-BODY GREEN's FUNCTIONS}

We work with a system of $N$ nonrelativistic fermions interacting by means of $2 \mathrm{~B}$ and $3 \mathrm{~B}$ interactions. We divide the Hamiltonian into two parts, $\hat{H}=\hat{H}_{0}+\hat{H}_{1} \cdot \hat{H}_{0}=\hat{T}+\hat{U}$ is an unperturbed, one-body contribution. It is the sum of the kinetic term and an auxiliary one-body operator $\hat{U}$, which defines the reference state for the perturbative expansion $\left|\Phi_{0}^{N}\right\rangle$, on top of which correlations will be added. ${ }^{1}$ The second term of the Hamiltonian, $\hat{H}_{1}=-\hat{U}+\hat{V}+\hat{W}$, includes the interactions. $\hat{V}$ and $\hat{W}$ denote, respectively, the two- and three-body interaction operators. In a second-quantized framework, the full Hamiltonian reads

$$
\begin{aligned}
\hat{H}= & \sum_{\alpha} \varepsilon_{\alpha}^{0} a_{\alpha}^{\dagger} a_{\alpha}-\sum_{\alpha \beta} U_{\alpha \beta} a_{\alpha}^{\dagger} a_{\beta}+\frac{1}{4} \sum_{\substack{\alpha \gamma \\
\beta \delta}} V_{\alpha \gamma, \beta \delta} a_{\alpha}^{\dagger} a_{\gamma}^{\dagger} a_{\delta} a_{\beta} \\
& +\frac{1}{36} \sum_{\substack{\alpha \gamma \epsilon \\
\beta \delta \eta}} W_{\alpha \gamma \epsilon, \beta \delta \eta} a_{\alpha}^{\dagger} a_{\gamma}^{\dagger} a_{\epsilon}^{\dagger} a_{\eta} a_{\delta} a_{\beta}
\end{aligned}
$$

The Greek indices $\alpha, \beta, \gamma, \ldots$ label a complete set of SP states which diagonalize the unperturbed Hamiltonian $\hat{H}_{0}$, with eigenvalues $\varepsilon_{\alpha}^{0}$. $a_{\alpha}^{\dagger}$ and $a_{\alpha}$ are creation and annihilation operators for a particle in state $\alpha$. The matrix elements of the $1 \mathrm{~B}$ operator $\hat{U}$ are given by $U_{\alpha \beta}$. Equivalently, the matrix elements of the $2 \mathrm{~B}$ and $3 \mathrm{~B}$ forces are $V_{\alpha \gamma, \beta \delta}$ and $W_{\alpha \gamma \epsilon, \beta \delta \eta}$. In the following, we work with antisymmetrized matrix elements in both the $2 \mathrm{~B}$ and the $3 \mathrm{~B}$ sectors.

The main ingredient of our formalism is the $1 \mathrm{~B}$ GF, also called SP propagator or two-point GF, which provides a complete description of one-particle and one-hole excitations of the many-body system. More specifically, the SP propagator is defined as the expectation value of the time-ordered product of an annihilation and a creation operator in the Heisenberg picture:

$$
i \hbar G_{\alpha \beta}\left(t_{\alpha}-t_{\beta}\right)=\left\langle\Psi_{0}^{N}\left|\mathcal{T}\left[a_{\alpha}\left(t_{\alpha}\right) a_{\beta}^{\dagger}\left(t_{\beta}\right)\right]\right| \Psi_{0}^{N}\right\rangle,
$$

where $\left|\Psi_{0}^{N}\right\rangle$ is the interacting $N$-body ground state of the system. The time ordering operator brings operators with earlier times to the right, with the corresponding fermionic permutation sign. For $t_{\alpha}-t_{\beta}>0$, this results in the addition of a particle to the state $\beta$ at time $t_{\beta}$ and its removal from state $\alpha$ at time $t_{\alpha}$. Alternatively, for $t_{\beta}-t_{\alpha}>0$, the removal of a particle from state $\alpha$ occurs at time $t_{\alpha}$ and its addition to state $\beta$ at time $t_{\beta}$. These correspond, respectively, to the propagation of a particle or a hole excitation through the system. We can

\footnotetext{
${ }^{1}$ A typical choice in nuclear physics would be a Slater determinant of a single-particle harmonic oscillator or a Woods-Saxon wave function.
} 
also introduce the four-point and six-point GFs:

$$
\begin{aligned}
i \hbar & G_{\alpha \gamma, \beta \delta}^{4-\mathrm{pt}}\left(t_{\alpha}, t_{\gamma} ; t_{\beta}, t_{\delta}\right) \\
\quad & =\left\langle\Psi_{0}^{N}\left|\mathcal{T}\left[a_{\gamma}\left(t_{\gamma}\right) a_{\alpha}\left(t_{\alpha}\right) a_{\beta}^{\dagger}\left(t_{\beta}\right) a_{\delta}^{\dagger}\left(t_{\delta}\right)\right]\right| \Psi_{0}^{N}\right\rangle, \\
i \hbar & G_{\alpha \gamma \epsilon, \beta \delta \eta}^{6-\mathrm{pt}}\left(t_{\alpha}, t_{\gamma}, t_{\epsilon} ; t_{\beta}, t_{\delta}, t_{\eta}\right) \\
\quad & =\left\langle\Psi_{0}^{N}\left|\mathcal{T}\left[a_{\epsilon}\left(t_{\epsilon}\right) a_{\gamma}\left(t_{\gamma}\right) a_{\alpha}\left(t_{\alpha}\right) a_{\beta}^{\dagger}\left(t_{\beta}\right) a_{\delta}^{\dagger}\left(t_{\delta}\right) a_{\eta}^{\dagger}\left(t_{\eta}\right)\right]\right| \Psi_{0}^{N}\right\rangle .
\end{aligned}
$$

Physically, the interpretation of Eqs. (3) and (4) follows that of the two-point GF in Eq. (2). In these cases, more combinations of particle and hole excitations are encountered depending on the ordering of the several time arguments. Note also that these propagators provide access to all $2 \mathrm{~B}$ and $3 \mathrm{~B}$ observables. The extension to formal expressions for higher many-body GFs is straightforward.

In the following, we will consider propagators both in time representation, as defined above, or in energy representation. Note that, due to time-translation invariance, the $m$-point GF depends only on $m-1$ time differences or, equivalently, $m-1$ independent frequencies. Hence the Fourier transform to the energy representation is only well defined when the total energy is conserved:

$$
\begin{aligned}
2 \pi & \delta\left(\omega_{\alpha}+\omega_{\gamma}+\cdots-\omega_{\beta}-\omega_{\delta}-\cdots\right) \\
& \times G_{\alpha \gamma \ldots, \beta \delta \ldots}^{m-\mathrm{pt}}\left(\omega_{\alpha}, \omega_{\gamma}, \ldots ; \omega_{\beta}, \omega_{\delta}, \ldots\right) \\
= & \int d t_{\alpha} \int d t_{\gamma} \cdots \int d t_{\beta} \int d t_{\delta} \cdots e^{i\left(\omega_{\alpha} t_{\alpha}+\omega_{\gamma} t_{\gamma}+\cdots\right)} \\
& \times G_{\alpha \gamma \ldots, \beta \delta \ldots}^{m-\mathrm{pt}}\left(t_{\alpha}, t_{\gamma}, \ldots ; t_{\beta}, t_{\delta}, \ldots\right) e^{-i\left(\omega_{\beta} t_{\beta}+\omega_{\delta} t_{\delta}+\cdots\right)} .
\end{aligned}
$$

For the 1B GFs, one also considers

$$
G_{\alpha \beta}(\omega)=\int d \tau e^{i \omega \tau} G_{\alpha \beta}(\tau)=G_{\alpha \beta}^{2-p t}(\omega, \omega) .
$$

Interactions in the many-body system can be treated by means of a perturbative expansion. For the $1 \mathrm{~B}$ propagator $G$, this expansion reads $[3,4]$

$$
\begin{aligned}
& G_{\alpha \beta}\left(t_{\alpha}-t_{\beta}\right)=-\frac{i}{\hbar} \sum_{n=0}^{\infty}\left(-\frac{i}{\hbar}\right)^{n} \frac{1}{n !} \int d t_{1} \ldots \int d t_{n} \\
& \times\left\langle\Phi_{0}^{N}\left|\mathcal{T}\left[\hat{H}_{1}\left(t_{1}\right) \cdots \hat{H}_{1}\left(t_{n}\right) a_{\alpha}^{I}\left(t_{\alpha}\right) a_{\beta}^{I^{\dagger}}\left(t_{\beta}\right)\right]\right| \Phi_{0}^{N}\right\rangle_{\mathrm{conn}}
\end{aligned}
$$

where $\left|\Phi_{0}^{N}\right\rangle$ is the unperturbed many-body ground state, i.e., our reference $N$-body state. $a_{\alpha}^{I}$ and $a_{\beta}^{I^{\dagger}}$ are now operators in the interaction picture with respect to $H_{0}$. The subscript "conn" implies that only connected diagrams have to be considered when performing the Wick contractions of the time-ordered product.

$H_{1}$ contains contributions from $1 \mathrm{~B}, 2 \mathrm{~B}$, and $3 \mathrm{~B}$ interactions. Thus, the expansion involves terms with individual contributions of each force, as well as combinations of these. Feynman diagrams are essential to keep track of such a variety of different contributions. The set of Feynman diagrammatic rules that stems out of Eq. (7) in the presence of $3 \mathrm{~B}$ interactions is reviewed in detail in Appendix A. In general, these are unchanged with respect to the $2 \mathrm{~B}$ case. However, we provide a few examples to illustrate the appearance of nontrivial symmetry factors when $3 \mathrm{~B}$ are considered. This complicates the rules of the symmetry factors and illustrates some of the difficulties associated with many-particle interactions. In the following, we will work mostly with unlabeled Feynman diagrams. We also work with antisymmetrized matrix elements but, in contrast to Hugenholtz diagrams [51], we expand the interaction vertices and show them with different types of lines for clarity.

A first reorganization of the contributions generated by Eq. (7) is obtained by considering one-particle reducible diagrams, i.e., diagrams that can be disconnected by cutting a single fermionic line. Reducible diagrams are generated by an all-orders summation through Dyson's equation [3]:

$$
G_{\alpha \beta}(\omega)=G_{\alpha \beta}^{(0)}(\omega)+\sum_{\gamma \delta} G_{\alpha \gamma}^{(0)}(\omega) \Sigma_{\gamma \delta}^{\star}(\omega) G_{\delta \beta}(\omega) \text {. }
$$

Thus, in practice, one only needs to include one-particle irreducible (1PI) contributions to the self-energy $\Sigma^{\star}$. The uncorrelated SP propagator $G^{(0)}$ is associated with the system governed by the $H_{0}$ Hamiltonian and represents the $n=0$ order in the expansion of Eq. (7). In the previous equation, $\omega$ corresponds to the energy of the propagating particle or hole excitation. The irreducible self-energy $\Sigma^{\star}$, appearing in Eq. (8), describes the kernel of all 1PI diagrams. This operator plays a central role in the GF formalism and can be interpreted as the nonlocal and energy-dependent interaction that each fermion feels due to the interaction with the medium. At positive energies, $\Sigma^{\star}(\omega)$ is also identified with the optical potential for scattering of a particle from the many-body target [51-55].

A further level of simplification in the self-energy expansion can be obtained if unperturbed propagators, $G^{(0)}$, in the internal fermionic lines are replaced by dressed GFs, $G$. This process is generically called propagator renormalization and further restricts the set of diagrams to skeleton diagrams [3,51]. These are defined as 1PI diagrams that do not contain any portion that can be disconnected by cutting a fermion line twice at any two different points. These portions would correspond to self-energy insertions, which are already re-summed into the dressed propagator $G$ by Eq. (8). The SCGF approach is precisely based on diagrammatic expansions of such skeleton diagrams with renormalized propagators.

In principle, this framework offers great advantages. First, it is intrinsically nonperturbative and completely independent from any choice of the reference state and auxiliary $1 \mathrm{~B}$ potential $\hat{U}$, which automatically drops out of Eq. (8). Second, many-body correlations are expanded directly in terms of SP excitations which are closer to the exact solution than those associated with the unperturbed state, $\left|\Phi_{0}^{N}\right\rangle$. Third, the number of diagrams is vastly reduced to 1PI skeletons. Fourth, a full SCGF calculation automatically satisfies the basic conservation laws $[3,56,57]$. In practice, however, calculating diagrams with dressed propagators is computationally more expensive than using the plain $G^{(0)}$ in perturbation theory. Moreover, self-consistency requires an iterative solution for $\Sigma^{\star}$ and for $G$ via the Dyson equation [Eq. (8)]. Therefore, the SCGF scheme is not always applied in full detail, but it is often employed to provide important guidance in developing working approximations to the self-energy. 


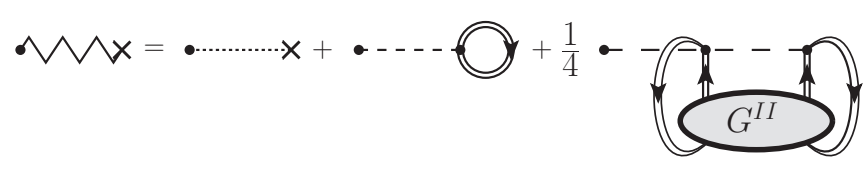

FIG. 1. Diagrammatic representation of the effective 1B interaction of Eq. (10). This is given by the sum of the original $1 \mathrm{~B}$ potential (dotted line), the $2 \mathrm{~B}$ interaction (dashed line) contracted with a dressed SP propagator $G$ (double line with arrow), and the $3 \mathrm{~B}$ interaction (long-dashed line) contracted with a dressed $2 \mathrm{~B}$ propagator $G^{I I}$. The correct symmetry factor of $1 / 4$ in the last term is also shown explicitly.

\section{A. Interaction-irreducible diagrams}

It is possible to further restrict the set of relevant diagrams by exploiting the concept of effective interactions. Let us consider an articulation vertex in a generic Feynman diagram. A $2 \mathrm{~B}, 3 \mathrm{~B}$ or higher interaction vertex is an articulation vertex if, when cut, it gives rise to two disconnected diagrams. ${ }^{2}$ Formally, a diagram is said to be interaction-irreducible if it contains no articulation vertices. Equivalently, a diagram is interaction reducible if there exists a group of fermion lines (either interacting or not) that leaves one interaction vertex and eventually all return to it.

When an articulation vertex is cut, one is left with a cycle of fermion lines that all connect to the same interaction. If there were $p$ lines connected to this interaction vertex, this set of closed lines would necessarily be part of a $2 p$-point $\mathrm{GF}^{3}$ If this GF is computed explicitly in the calculation, one can use it to evaluate all these contributions straight away. This eliminates the need for computing all the diagrams looping in and out of the articulation vertex, at the expense of having to find the many-body propagator. An $n$-body interaction vertex with $p$ fermion lines looping over it is an $n-p$ effective interaction operator. Infinite sets of interaction-reducible diagrams can be subsummed by means of effective interactions.

The two cases of interest when $2 \mathrm{~B}$ and $3 \mathrm{~B}$ forces are present in the Hamiltonian are shown in Figs. 1 and 2 that give, respectively, the diagrammatic definition of the $1 \mathrm{~B}$ and $2 \mathrm{~B}$ effective interactions. The $1 \mathrm{~B}$ effective interaction is obtained by adding up three contributions: the original $1 \mathrm{~B}$ interaction; a $1 \mathrm{~B}$ average over the $2 \mathrm{~B}$ interaction; and a $2 \mathrm{~B}$ average over the $3 \mathrm{~B}$ force. The $1 \mathrm{~B}$ and $2 \mathrm{~B}$ averages are performed using fully dressed propagators. Similarly, an effective $2 \mathrm{~B}$ force is obtained from the original $2 \mathrm{~B}$ interaction plus a $1 \mathrm{~B}$ average over the $3 \mathrm{~B}$ force. Note that these go beyond usual normal-ordering "averages" in that they are performed over fully correlated, many-body propagators. Similar definitions would hold for higher-order forces and effective interactions beyond the 3B level.

Hence, for a system with up to $3 \mathrm{BF}$, we define an effective Hamiltonian,

$$
\widetilde{H}_{1}=\widetilde{U}+\widetilde{V}+\hat{W}
$$

\footnotetext{
${ }^{2} 1 \mathrm{~B}$ vertices cannot be split and therefore cannot be articulations.

${ }^{3}$ More specifically, these fermion lines contain an instantaneous contribution of the many-body GF that enters and exits the same interaction vertex, corresponding to a $p$-body reduced density matrix.
}

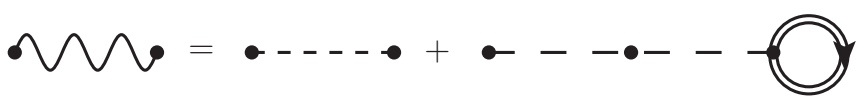

FIG. 2. Diagrammatic representation of the effective 2B interaction of Eq. (11). This is given by the sum of the original $2 \mathrm{~B}$ interaction (dashed line) and the 3B interaction (long-dashed line) contracted with a dressed SP propagator $G$.

where $\widetilde{U}$ and $\widetilde{V}$ represent effective interaction operators. The diagrammatic expansion arising from Eq. (7) with the effective Hamiltonian $\widetilde{H}_{1}$ is formed only of (1PI, skeleton) interaction-irreducible diagrams to avoid any possible double counting. Note that the $3 \mathrm{~B}$ interaction $\hat{W}$ remains the same as in Eq. (1) but enters only the interaction-irreducible diagrams with respect to $3 \mathrm{~B}$ interactions. The explicit expressions for the $1 \mathrm{~B}$ and $2 \mathrm{~B}$ effective interaction operators are

$$
\begin{aligned}
\widetilde{U}= & \sum_{\alpha \beta}\left[-U_{\alpha \beta}-i \hbar \sum_{\gamma \delta} V_{\alpha \gamma, \beta \delta} G_{\delta \gamma}\left(t-t^{+}\right)\right. \\
& \left.+\frac{i \hbar}{4} \sum_{\substack{\gamma \epsilon \\
\delta \eta}} W_{\alpha \gamma \epsilon, \beta \delta \eta} G_{\delta \eta, \gamma \epsilon}^{I I}\left(t-t^{+}\right)\right] a_{\alpha}^{\dagger} a_{\beta}, \\
\widetilde{V}= & \frac{1}{4} \sum_{\substack{\alpha \gamma \\
\beta \delta}}\left[V_{\alpha \gamma, \beta \delta}-i \hbar \sum_{\epsilon \eta} W_{\alpha \gamma \epsilon, \beta \delta \eta} G_{\eta \epsilon}\left(t-t^{+}\right)\right] a_{\alpha}^{\dagger} a_{\gamma}^{\dagger} a_{\delta} a_{\beta} .
\end{aligned}
$$

We have introduced a specific component of the four-point GFs,

$$
G_{\delta \eta, \gamma \epsilon}^{I I}\left(t-t^{\prime}\right)=G_{\delta \eta, \gamma \epsilon}^{4-\mathrm{pt}}\left(t^{+}, t ; t^{\prime}, t^{\prime+}\right),
$$

which involves two-particle and two-hole propagation. This is the so-called two-particle and two-time Green's function. Let us also note that the contracted propagators in Eqs. (10) and (11) correspond to the full $1 \mathrm{~B}$ and $2 \mathrm{~B}$ reduced density matrices of the many-body system:

$$
\begin{aligned}
\rho_{\delta \gamma}^{1 B} & =\left\langle\Psi_{0}^{N}\left|a_{\gamma}^{\dagger} a_{\delta}\right| \Psi_{0}^{N}\right\rangle=-i \hbar G_{\delta \gamma}\left(t-t^{+}\right), \\
\rho_{\delta \eta, \gamma \epsilon}^{2 B} & =\left\langle\Psi_{0}^{N}\left|a_{\gamma}^{\dagger} a_{\epsilon}^{\dagger} a_{\eta} a_{\delta}\right| \Psi_{0}^{N}\right\rangle=i \hbar G_{\delta \eta, \gamma \epsilon}^{I I}\left(t-t^{+}\right) .
\end{aligned}
$$

In a self-consistent calculation, effective interactions should be computed iteratively at each step, using correlated $1 \mathrm{~B}$ and 2B propagators as input.

The effective Hamiltonian of Eq. (9) not only regroups Feynman diagrams in a more efficient way, but also defines the effective $1 \mathrm{~B}$ and $2 \mathrm{~B}$ terms from higher order interactions. Averaging the $3 \mathrm{BF}$ over one and two spectator particles in the medium is expected to yield the most important contributions to the many-body dynamics in nuclei $[31,33]$. We note that Eqs. (10) and (11) are exact and can be derived rigorously from the perturbative expansion. Details of the proof are discussed in Appendix B. As long as interaction-irreducible diagrams are used together with the effective Hamiltonian $\widetilde{H}_{1}$, this approach provides a systematic way to incorporate many-body forces in the calculations and to generate effective in-medium interactions. More importantly, the formalism is 
such that symmetry factors are properly considered and no diagram is overcounted.

This approach can be seen as a generalization of the normal ordering of the Hamiltonian with respect to the reference state $\left|\Phi_{0}^{N}\right\rangle$, a procedure that is already used in nuclear physics applications with 3BFs $[31,33,48]$. In both the traditional normal ordering and our approach, the $\widetilde{U}$ and $\widetilde{V}$ operators contain contributions from higher order forces, while $\hat{W}$ remains unchanged. The normal ordered interactions affect only excited configurations with respect to $\left|\Phi_{0}^{N}\right\rangle$, but not the reference state itself. Similarly, the effective operators discussed above only enter interaction-irreducible diagrams. As a matter of fact, if the unperturbed $1 \mathrm{~B}$ and $2 \mathrm{~B}$ propagators were used in Eqs. (10) and (11), the effective operators $\widetilde{U}$ and $\widetilde{V}$ would trivially reduce to the contracted $1 \mathrm{~B}$ and $2 \mathrm{~B}$ terms of normal ordering. In the present case, however, the contraction goes beyond normal ordering because it is performed with respect to the exact correlated density matrices. To some extent, one can think of the effective Hamiltonian $\widetilde{H}$ as being reordered with respect to the interacting many-body ground state $\left|\Psi_{0}^{N}\right\rangle$, rather than the noninteracting $\left|\Phi_{0}^{N}\right\rangle$. This effectively incorporates correlations that, in the normal ordering procedure, must be instead calculated explicitly by the many-body approach. Calculations indicate that such correlated averages are important in both the saturation mechanism of nuclei and nuclear matter [27,35].

Note that a normal ordered Hamiltonian also contains a $0 \mathrm{~B}$ term equal to the expectation value of the original Hamiltonian $\hat{H}$, with respect to $\left|\Phi_{0}^{N}\right\rangle$. Likewise, the full contraction of $\hat{H}$, according to the procedure of Appendix B, will yield the exact ground-state energy:

$$
\begin{aligned}
E_{0}^{N}= & -i \hbar \sum_{\alpha \beta} T_{\alpha \beta} G_{\beta \alpha}\left(t-t^{+}\right) \\
& +\frac{i \hbar}{4} \sum_{\substack{\alpha \gamma \\
\beta \delta}} V_{\alpha \gamma, \beta \delta} G_{\beta \delta, \alpha \gamma}^{I I}\left(t-t^{+}\right) \\
& -\frac{i \hbar}{36} \sum_{\substack{\alpha \gamma \epsilon \\
\beta \delta \eta}} W_{\alpha \gamma \epsilon, \beta \delta \eta} G_{\beta \delta \eta, \alpha \gamma \epsilon}^{I I I}\left(t-t^{+}\right) \\
= & \left\langle\Psi_{0}^{N}|H| \Psi_{0}^{N}\right\rangle,
\end{aligned}
$$

in accordance with our analogy between the effective Hamiltonian, $\widetilde{H}=\hat{H}_{0}+\widetilde{H}_{1}$, and the usual normal ordering.

Before we move on, let us mention a subtlety arising in the Hartree-Fock (or lowest-order) approximation to the two-body propagator. If one were to insert $\widetilde{V}$ into the second term of the right-hand side of Eq. (10), one would introduce a double counting of the pure $3 \mathrm{BF}$ Hartree-Fock component. This is forbidden because the diagram in question would be interaction reducible. The correct $3 \mathrm{BF}$ Hartree-Fock term is actually included as part of the last term of Eq. (10) (see also Fig. 1). Consequently, there is no Hartree-Fock term arising from the effective interactions. Instead, this lowest-order contribution is fully taken into account within the $1 \mathrm{~B}$ effective interaction.

\section{B. Self-energy expansion up to third order}

As a demonstration of the simplification introduced by the effective interaction approach, in this subsection we will derive all interaction-irreducible contributions to the proper self-energy up to third order in perturbation theory. We will discuss these contributions order by order, thus providing an overview of how the approach can be extended to higher-order perturbative and also to nonperturbative calculations. Among other things, this exercise will illustrate the amount and variety of new diagrams that need to be considered when $3 \mathrm{BFs}$ are used.

For a pure 2B Hamiltonian, the only possible interactionreducible contribution to the self-energy is the generalized Hartree-Fock diagram. This corresponds to the second term on the right-hand side of Eq. (10) (see also Fig. 1). Note that this can go beyond the usual Hartree-Fock term in that the internal propagator is dressed. This diagram appears at first order in any SCGF expansion and it is routinely included in most GF calculations with $2 \mathrm{~B}$ forces. Thus, regrouping diagrams in terms of effective interactions, such as Eqs. (10) and (11), give no practical advantages unless 3BFs (or higher-body forces) are present.

If $3 \mathrm{BFs}$ are considered, the only first-order, interactionirreducible contribution is precisely given by the one-body effective interaction depicted in Fig. 1,

$$
\Sigma_{\alpha \beta}^{\star,(1)}=\widetilde{U}_{\alpha \beta}
$$

Because $\widetilde{U}$ is in itself a self-energy insertion, it will not appear in any other, higher-order skeleton diagram. Even though it only contributes to Eq. (16), the effective $1 \mathrm{~B}$ potential is very important because it determines the energy-independent part of the self-energy. It therefore represents the (static) mean field seen by every particle, due to both $2 \mathrm{~B}$ and 3B interactions. As already mentioned, Eq. (10) shows that this potential incorporates three separate terms, including the Hartree-Fock potentials due to both $2 \mathrm{~B}$ and $3 \mathrm{BFs}$ and higher-order, interaction-reducible contributions due to the dressed $G$ and $G^{I I}$ propagators. Thus, even the calculation of this lowest-order term $\Sigma^{\star,(1)}$ requires an iterative procedure to evaluate the internal many-body propagators self-consistently.

Note that, if one were to stop at the Hartree-Fock level, the four-point GF would reduce to the direct and exchange product of two 1B propagators. In that case, the last term of Eq. (10) (or Fig. 1) would reduce to the pure 3BF Hartree-Fock contribution with the correct $1 / 2$ factor in front, due to the two equivalent fermionic lines. This approximate treatment of the $2 \mathrm{~B}$ propagator in the $1 \mathrm{~B}$ effective interaction was employed in most nuclear physics calculations up to date, including both finite nuclei [32,33,35] and nuclear matter [25-27,37,58,59] applications. Numerical implementations of averages with fully correlated $2 \mathrm{~B}$ propagators are underway.

At second order, there are only two interaction-irreducible diagrams, that we show in Fig. 3. Diagram Fig. 3(a) has the same structure as the well-known contribution due to 2BFs only, involving two-particle-one-hole $(2 p 1 h)$ and twohole-one-particle $(2 h 1 p)$ intermediate states. This diagram, however, is computed with the $2 \mathrm{~B}$ effective interaction (notice the wiggly line) instead of the original $2 \mathrm{~B}$ force and hence 


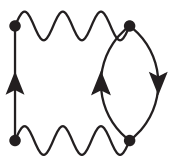

(a)

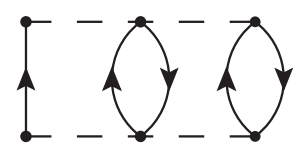

(b)
FIG. 3. 1PI, skeleton and interaction-irreducible self-energy diagrams appearing at second order in the perturbative expansion of Eq. (7), using the effective Hamiltonian of Eq. (9).

it corresponds to further interaction-reducible diagrams. By expanding the effective $2 \mathrm{~B}$ interaction according to Eq. (11), the contribution of Fig. 3(a) splits into the four diagrams of Fig. 4 (see also a similar example in Fig. 16).

The second interaction-irreducible diagram arises from explicit 3BFs and it is given in Fig. 3(b). One may expect this contribution to play a minor role due to phase space arguments, as it involves $3 p 2 h$ and $3 h 2 p$ excitations at higher excitation energies. Moreover, 3BFs are generally weaker than the corresponding $2 \mathrm{BF}$ (typically, $\langle\widehat{W}\rangle \approx \frac{1}{10}\langle\widehat{V}\rangle$ for nuclear interactions $[22,46])$. Summarizing, at second order in standard self-consistent perturbation theory, one would find a total of five skeleton diagrams. Of these, only two are interaction irreducible and need to be calculated when effective interactions are considered.

Figure 5 shows all the 17 interaction-irreducible diagrams appearing at third order. Again, note that, expanding the effective interaction $\widetilde{V}$, would generate a much larger number of diagrams (53 in total). Diagrams Figs. 5(a) and 5(b) are the only third-order terms that would appear in the $2 \mathrm{BF}$ case. Numerically, these two diagrams only require evaluating Eq. (11) beforehand, but can otherwise be dealt with using existing $2 \mathrm{BF}$ codes. They have already been exploited to include 3BFs in nuclear structure studies [21,25,27,35,37].

The remaining 15 diagrams, from Figs. 5(c)-5(q), appear when 3BFs are introduced. These third-order diagrams are ordered in Fig. 5 in terms of increasing numbers of $3 \mathrm{~B}$ interactions and, within these, in terms of increasing number of particle-hole excitations. Qualitatively, one would expect that this should correspond to a decreasing importance of their contributions. Diagrams Figs. 5(a)-5(c), for instance, only involve $2 p 1 h$ and $2 h 1 p$ intermediate configurations, normally

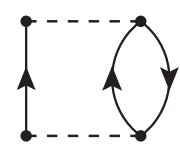

(a)

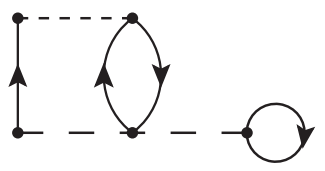

(c)

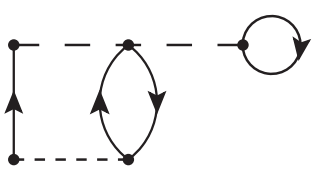

(b)

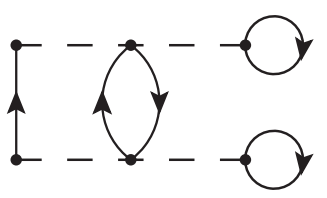

(d)
FIG. 4. These four diagrams are contained in diagram Fig. 3(a). They correspond to one $2 \mathrm{~B}$ interaction-irreducible diagram (a), and three interaction-reducible diagrams (b)-(d). needed to describe particle addition and removal energies to dominant quasiparticle peaks as well as total ground-state energies.

Diagram Fig. 5(c) includes one 3B irreducible interaction term and still needs to be investigated within the SCGF method. Normal-ordered Hamiltonian studies [31,33] clearly suggest that this brings in a small correction to the total energy with respect to diagrams Figs. 5(a) and 5(b). This is in line with the qualitative analysis of the number of $\widetilde{V}$ and $\hat{W}$ interactions entering these diagrams. Diagrams Figs. 5(a)-5(c) all represent the first-order term in an all-order summation needed to account for configuration mixing between $2 p 1 h$ or $2 h 1 p$ excitations. Nowadays, resummations of these configurations are performed routinely for the first two diagrams in third-order algebraic diagrammatic construction, $\operatorname{ADC}(3)$, and FRPA calculations $[10,11,16]$.

The remaining diagrams of Fig. 5 all include $3 p 2 h$ and $3 h 2 p$ configurations. These become necessary to reproduce the fragmentation patterns of shakeup configurations in particle removal and addition experiments, i.e., Dyson orbits beyond the main quasiparticle peaks. These contributions are computationally more demanding. Diagrams Figs. 5(d)-5(k) all describe interaction between $2 p 1 h(2 h 1 p)$ and $3 p 2 h$ $(3 h 2 p)$ configurations. These are split into four contributions arising from two effective $2 \mathrm{BFs}$ and four that contain two irreducible 3B interactions. Similarly, diagrams Figs. 5(1)-5(q) are the first contributions to the configuration mixing among $3 p 2 h$ or $3 h 2 p$ states.

Appendix A provides the Feynman diagram rules to compute the contribution associated with these diagrams. Specific expressions for some diagrams in Fig. 5 are given. We note that the Feynman rules remain unaltered whether one uses the original, $\hat{U}$ and $\hat{V}$, or the effective, $\widetilde{U}$ or $\widetilde{V}$, interactions. Hence, symmetry factors from equivalent lines remain unchanged.

\section{EQUATION-OF-MOTION METHOD}

The perturbation theory expansion outlined in the previous section is useful to identify new contributions arising from the inclusion of $3 \mathrm{~B}$ interactions. However, diagrams up to third order alone do not necessarily incorporate all the necessary information to describe strongly correlated quantum manybody systems. For example, the strong repulsive character of the nuclear force at short distances requires explicit allorder summations of ladder series. All-order summations of $2 p 1 h$ and $2 h 1 p$ are also required in finite systems to achieve accuracy for the predicted ground-state and separation energies, as well as to preserve the correct analytic properties of the self-energy beyond second order.

To investigate approximation schemes for all-order summations including 3BFs, we now develop the EOM method. This will provide special insight into possible self-consistent expansions of the irreducible self-energy, $\Sigma^{\star}$. For $2 \mathrm{~B}$ forces only, the EOM technique defines a hierarchy of equations that link each $n$-body GF to the $(n-1)$ - and the $(n+1)$-body GFs. When extended to include $3 \mathrm{BF}$, the hierarchy also involves the $(n+2)$-body GFs. A truncation of this Martin-Schwinger hierarchy is necessary to solve the system of equations [5] and 


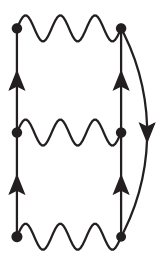

(a)

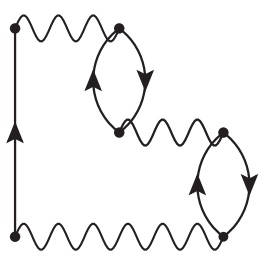

(b)

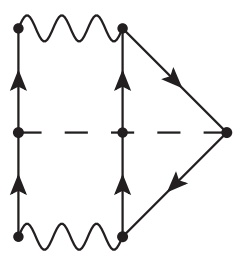

(c)

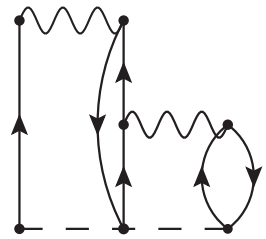

(d)

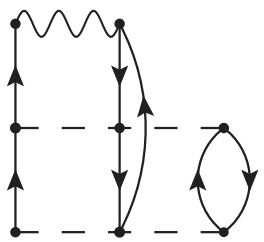

(h)

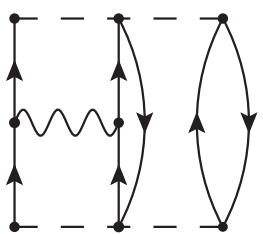

(1)

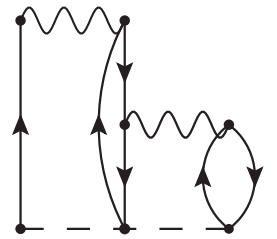

(e)

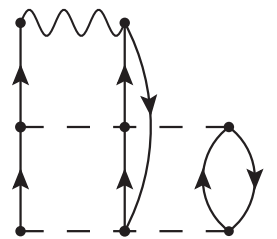

(i)

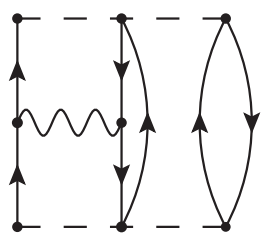

(m)

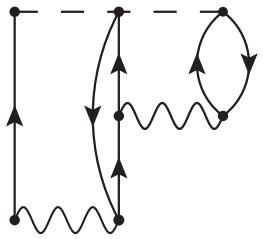

(f)

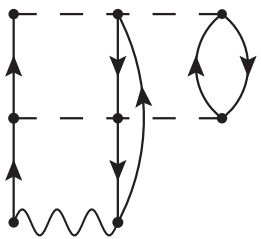

(j)

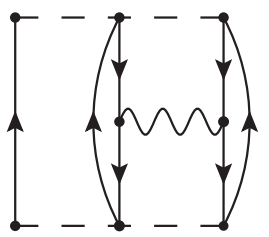

(n)

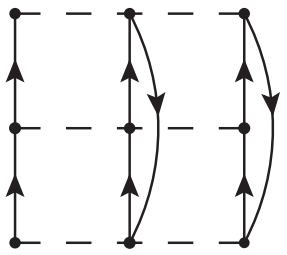

(o)

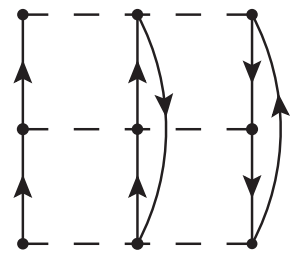

(p)

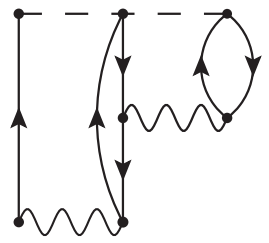

(g)

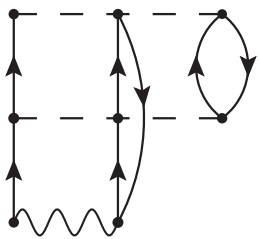

(k)

FIG. 5. 1PI, skeleton and interaction-irreducible self-energy diagrams appearing at third order in the perturbative expansion of Eq. (7) using the effective Hamiltonian of Eq. (9).

can potentially give rise to physically relevant resummation schemes. Here, we will follow the footprints of Ref. [60] and apply truncations to obtain explicit equations for the four-point (and six-point, in the 3BF case) vertex functions.

\section{A. Equation of motion for $G$ and proper self-energy}

The EOM for a given propagator is found by taking the derivative of its time arguments. The time arguments are linked to the creation and annihilation operators in Eqs. (2)-(4) and hence the time dependence of these operators will drive that of the propagator [51]. The unperturbed 1B propagator can be written as the $n=0$ order term of Eq. (7),

$$
i \hbar G_{\alpha \beta}^{(0)}\left(t_{\alpha}-t_{\beta}\right)=\left\langle\Phi_{0}^{N}\left|\mathcal{T}\left[a_{\alpha}^{I}\left(t_{\alpha}\right) a_{\beta}^{I^{\dagger}}\left(t_{\beta}\right)\right]\right| \Phi_{0}^{N}\right\rangle .
$$

Its time derivative will be given by the von Neumann equation for the operators in the interaction picture [6]:

$$
i \hbar \frac{\partial}{\partial t} a_{\alpha}^{I}(t)=\left[a_{\alpha}^{I}(t), \hat{H}_{0}\right]=\varepsilon_{\alpha}^{0} a_{\alpha}^{I}(t) .
$$


Taking the derivative of $G^{(0)}$ with respect to time and using Eq. (18), we find

$$
\left\{i \hbar \frac{\partial}{\partial t_{\alpha}}-\varepsilon_{\alpha}^{0}\right\} G_{\alpha \beta}^{(0)}\left(t_{\alpha}-t_{\beta}\right)=\delta\left(t_{\alpha}-t_{\beta}\right) \delta_{\alpha \beta}
$$

Note that the delta functions in time arise from the derivatives of the step functions involved in the time-ordered product.

The same procedure applied to the exact 1B propagator $G$ requires the time derivative of the operators in the Heisenberg picture. For the original Hamiltonian of Eq. (1), the EOM for the annihilation operator reads

$$
\begin{aligned}
i \hbar \frac{\partial}{\partial t} a_{\alpha}(t)= & {\left[a_{\alpha}(t), \hat{H}\right]=\varepsilon_{\alpha}^{0} a_{\alpha}(t)-\sum_{\delta} U_{\alpha \delta} a_{\delta}(t) } \\
& +\frac{1}{2} \sum_{\substack{\epsilon \mu \\
\delta \mu}} V_{\alpha \epsilon, \delta \mu} a_{\epsilon}^{\dagger}(t) a_{\mu}(t) a_{\delta}(t) \\
& +\frac{1}{12} \sum_{\substack{\epsilon \theta \\
\delta \mu \lambda}} W_{\alpha \in \theta, \delta \mu \lambda} a_{\epsilon}^{\dagger}(t) a_{\theta}^{\dagger}(t) a_{\lambda}(t) a_{\mu}(t) a_{\delta}(t) .
\end{aligned}
$$

This can now be used to take the derivative of the full 1B propagator in Eq. (2):

$$
\begin{aligned}
& \left\{i \hbar \frac{\partial}{\partial t_{\alpha}}-\varepsilon_{\alpha}^{0}\right\} G_{\alpha \beta}\left(t_{\alpha}-t_{\beta}\right) \\
& =\delta\left(t_{\alpha}-t_{\beta}\right) \delta_{\alpha \beta}-\sum_{\delta} U_{\alpha \delta} G_{\delta \beta}\left(t_{\alpha}-t_{\beta}\right) \\
& \quad+\frac{1}{2} \sum_{\epsilon} V_{\alpha \epsilon, \delta \mu} G_{\delta \mu, \epsilon \beta}^{4-\mathrm{pt}}\left(t_{\alpha}, t_{\alpha} ; t_{\alpha}^{+}, t_{\beta}\right) \\
& \quad+\frac{1}{12} \sum_{\delta \mu} W_{\alpha \epsilon \theta, \delta \mu \lambda} G_{\delta \mu \lambda, \epsilon \theta \beta}^{6-\mathrm{pt}}\left(t_{\alpha}, t_{\alpha}, t_{\alpha} ; t_{\alpha}^{++}, t_{\alpha}^{+}, t_{\beta}\right) .
\end{aligned}
$$

This equation links the two-point GF to both the four- and the six-point GFs. Note that the connection with the latter is mediated by the 3BF and hence does not appear in the pure $2 \mathrm{BF}$ case. Regarding the time arguments in Eq. (21), the $t_{\alpha}^{+}$ and $t_{\alpha}^{++}$present in the four- and six-point GFs are necessary to keep the correct time ordering in the creation operators when going from Eq. (20) to Eq. (21). An analogous equation can be obtained for the derivative of the time argument $t_{\beta}$. After some manipulation, involving the Fourier transforms of Eqs. (5) and (6), one obtains the equation of motion for the SP propagator in frequency representation:

$$
\begin{aligned}
G_{\alpha \beta}(\omega)= & G_{\alpha \beta}^{(0)}(\omega)-\sum_{\gamma \delta} G_{\alpha \gamma}^{(0)}(\omega) U_{\gamma \delta} G_{\delta \beta}(\omega)-\frac{1}{2} \sum_{\substack{\gamma \epsilon \\
\delta \mu}} G_{\alpha \gamma}^{(0)}(\omega) V_{\gamma \epsilon, \delta \mu} \int \frac{d \omega_{1}}{2 \pi} \int \frac{d \omega_{2}}{2 \pi} G_{\delta \mu, \beta \epsilon}^{4-\mathrm{pt}}\left(\omega_{1}, \omega_{2} ; \omega, \omega_{1}+\omega_{2}-\omega\right) \\
& +\frac{1}{12} \sum_{\substack{\gamma \epsilon \theta \\
\delta \mu \lambda}} G_{\alpha \epsilon}^{(0)}(\omega) W_{\gamma \epsilon \theta, \delta \mu \lambda} \int \frac{d \omega_{1}}{2 \pi} \int \frac{d \omega_{2}}{2 \pi} \int \frac{d \omega_{3}}{2 \pi} \int \frac{d \omega_{4}}{2 \pi} G_{\delta \mu \lambda, \gamma \beta \theta}^{6-\mathrm{pt}}\left(\omega_{1}, \omega_{2}, \omega_{3} ; \omega_{4}, \omega, \omega_{1}+\omega_{2}+\omega_{3}-\omega_{4}-\omega\right) .
\end{aligned}
$$

Again, this involves both the four- and the six-point GFs, which appear because of the $2 \mathrm{~B}$ and $3 \mathrm{~B}$ interactions, respectively. The equation now involves $n-2$ frequency integrals of the $n$-point GFs. The diagrammatic representation of this equation is given in Fig. 6 .
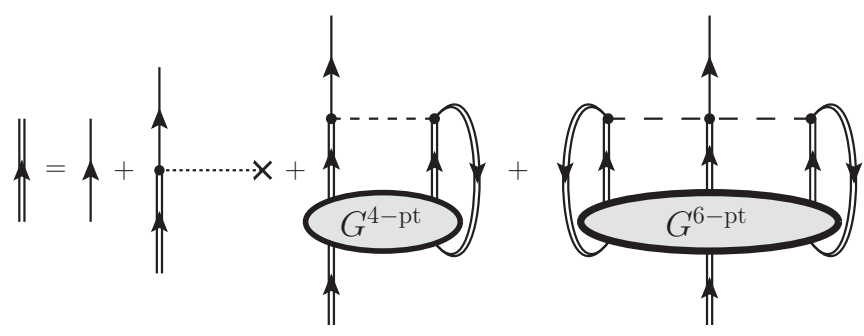

FIG. 6. Diagrammatic representation of the EOM, Eq. (22), for the dressed $1 \mathrm{~B}$ propagator $G$. The first term, given by a single line, defines the free $1 \mathrm{~B}$ propagator $G^{(0)}$. The second term denotes the interaction with a bare $1 \mathrm{~B}$ potential, whereas the third and the fourth terms describe interactions involving the intermediate propagation of two- and three-particle configurations.
The EOMs, Eqs. (21) and (22), connect the 1B propagator to GFs of different orders. In general, starting from an $n$-body GF, the derivative of the time-ordering operator generates a delta function between an incoming and outgoing particle, effectively separating a line and leaving an $(n-1)$-body propagator. Conversely, the $2 \mathrm{~B}$ part of the Hamiltonian introduces an extra pair of creation and annihilation operators that adds another particle and leads to an $(n+1)$-body GF. For a 3B Hamiltonian, the $(n+2)$-body GF enters the EOM because of the commutator in Eq. (20). This implies that higher order GFs will be needed, at the same level of approximation, in the EOM hierarchy with 3BFs.

Equation (22) gives an exact equation for the SP propagator $G$ that, however, requires the knowledge of both the four-point and six-point GFs. Full equations for the latter require applying the EOMs to these propagators as well. Before that, however, it is possible to further simplify contributions in Eq. (22) by splitting the $n$-point GFs into two terms. The first one is relatively simple, involving the properly antisymmetrized independent propagation of $n$ dressed particles. The second term will involve the interaction vertices, $\Gamma^{4-\mathrm{pt}}$ and $\Gamma^{6-\mathrm{pt}}, 1 \mathrm{PI}$ 
vertex functions that include all interaction effects [51]. These can be neatly connected to the irreducible self-energy.

For the four-point GF, this separation is shown diagrammatically in Fig. 7. The first two terms involve two dressed fermion lines propagating independently, and their exchange as required by the Pauli principle. The remaining part, stripped of its external legs, can contain only 1PI diagrams which are collected in a vertex function, $\Gamma^{4-\mathrm{pt}}$. This is associated with interactions and, at lowest level, it would correspond to a $2 \mathrm{BF}$. As we will see in the following, however, 3B interactions also provide contributions to $\Gamma^{4-\mathrm{pt}}$. The four-point vertex function is defined by the following equation:

$$
\begin{aligned}
& G_{\alpha \gamma, \beta \delta}^{4-\mathrm{pt}}\left(\omega_{\alpha}, \omega_{\gamma} ; \omega_{\beta}, \omega_{\delta}\right) \\
& =\quad i \hbar\left[2 \pi \delta\left(\omega_{\alpha}-\omega_{\beta}\right) G_{\alpha \beta}\left(\omega_{\alpha}\right) G_{\gamma \delta}\left(\omega_{\gamma}\right)\right. \\
& \left.\quad-2 \pi \delta\left(\omega_{\gamma}-\omega_{\beta}\right) G_{\alpha \delta}\left(\omega_{\alpha}\right) G_{\gamma \beta}\left(\omega_{\gamma}\right)\right] \\
& +(i \hbar)^{2} \sum_{\substack{\theta \mu \\
\nu \lambda}} G_{\alpha \theta}\left(\omega_{\alpha}\right) G_{\gamma \mu}\left(\omega_{\gamma}\right) \\
& \quad \times \Gamma_{\theta \mu, \nu \lambda}^{4-\mathrm{pt}}\left(\omega_{\alpha}, \omega_{\gamma} ; \omega_{\beta}, \omega_{\delta}\right) G_{\nu \beta}\left(\omega_{\beta}\right) G_{\lambda \delta}\left(\omega_{\delta}\right) .
\end{aligned}
$$

Equation (23) is exact and is an implicit definition of $\Gamma^{4-p t}$. Different many-body approximations arise when approximations are performed on this vertex function $[3,14]$.

A similar expression holds for the six-point GF. In this case, the diagrams that involve noninteracting lines can contain either all three dressed propagators moving independently from each other or groups of two lines interacting through a four-point vertex function. The remaining terms are collected in a six-point vertex function, $\Gamma^{6-p t}$, which contains terms where all three lines are interacting. This separation is demonstrated diagrammatically in Fig. 8. The Pauli principle requires a complete antisymmetrization of these diagrams. For the "free propagating" term, this implies all $3 !=6$ permutations of the three lines. The second term, involving $\Gamma^{4-\mathrm{pt}}$, requires $3^{2}=9$ cyclic permutations within both incoming and outgoing legs. The six-point vertex function is already antisymmetrized and hence no permutations are needed.

The equation corresponding to Fig. 8 is exact and provides an implicit definition of the $\Gamma^{6-p t}$ vertex function:

$$
\begin{aligned}
& G_{\alpha \gamma \epsilon, \beta \delta \eta}^{6-\mathrm{pt}}\left(\omega_{\alpha}, \omega_{\gamma}, \omega_{\epsilon} ; \omega_{\beta}, \omega_{\delta}, \omega_{\eta}\right)=(2 \pi)^{2}(i \hbar)^{2} \mathcal{A}_{\left[\left\{\alpha \omega_{\alpha}\right\},\left\{\gamma \omega_{\gamma}\right\},\left\{\epsilon \omega_{\epsilon}\right\}\right]}\left[\delta\left(\omega_{\alpha}-\omega_{\beta}\right) \delta\left(\omega_{\gamma}-\omega_{\delta}\right) G_{\alpha \beta}\left(\omega_{\alpha}\right) G_{\gamma \delta}\left(\omega_{\gamma}\right) G_{\epsilon \eta}\left(\omega_{\epsilon}\right)\right] \\
& +2 \pi(i \hbar)^{3} \mathcal{P}_{\left[\left\{\alpha \omega_{\alpha}\right\},\left\{\gamma \omega_{\gamma}\right\},\left\{\epsilon \omega_{\epsilon}\right\}\right]}^{\text {cycl. }} \mathcal{P}_{\left[\left\{\beta \omega_{\beta}\right\},\left\{\delta \omega_{\delta}\right\},\left\{\eta \omega_{\eta}\right\}\right]}^{\text {cycl. }}\left[\delta\left(\omega_{\alpha}-\omega_{\beta}\right) G_{\alpha \beta}\left(\omega_{\alpha}\right)\right. \\
& \left.\times \sum_{\theta \mu} G_{\gamma \theta}\left(\omega_{\gamma}\right) G_{\epsilon \mu}\left(\omega_{\epsilon}\right) \Gamma_{\theta \mu, \nu \lambda}^{4-\mathrm{pt}}\left(\omega_{\gamma}, \omega_{\epsilon} ; \omega_{\delta}, \omega_{\eta}\right) G_{\nu \delta}\left(\omega_{\delta}\right) G_{\lambda \eta}\left(\omega_{\eta}\right)\right] \\
& +(i \hbar)^{4} \sum_{\substack{\theta \mu \chi \\
\nu \lambda \xi}} G_{\alpha \theta}\left(\omega_{\alpha}\right) G_{\gamma \mu}\left(\omega_{\gamma}\right) G_{\epsilon \chi}\left(\omega_{\epsilon}\right) \Gamma_{\theta \mu \chi, \nu \lambda \xi}^{6-p t}\left(\omega_{\alpha}, \omega_{\gamma}, \omega_{\epsilon} ; \omega_{\beta}, \omega_{\delta}, \omega_{\eta}\right) \\
& \times G_{\nu \beta}\left(\omega_{\beta}\right) G_{\lambda \delta}\left(\omega_{\delta}\right) G_{\xi \eta}\left(\omega_{\eta}\right) .
\end{aligned}
$$

Here, we have introduced the antisymmetrization operator $\mathcal{A}$, which sums all possible permutations of pairs of indices and

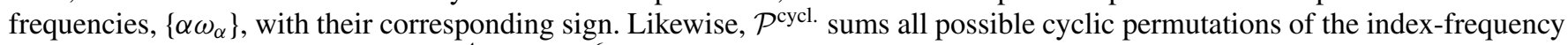
pairs. Again, let us stress that both $\Gamma^{4-\mathrm{pt}}$ and $\Gamma^{6-\mathrm{pt}}$ are formed of 1PI diagrams only because they are defined by removing all external dressed legs from the $G^{4-\mathrm{pt}}$ and $G^{6-\mathrm{pt}}$ propagators. However, they are still two-particle reducible, because they include diagrams that can be split by cutting two lines. In general, $\Gamma^{4-\mathrm{pt}}$ and $\Gamma^{6-\mathrm{pt}}$ are solution of all-order summations analogous to the Bethe-Salpeter equation, in which the kernels are 2PI and 3PI vertices [see Eqs. (27)-(29) below].

Inserting Eqs. (23) and (24) into Eq. (22), and exploiting the effective 1B and 2B operators defined in Eqs. (10) and (11), one recovers the Dyson equation [Eq. (8)]. One can therefore identify the exact expression of the irreducible self-energy $\Sigma^{\star}$ in terms of 1PI vertex functions:

$$
\begin{aligned}
\Sigma_{\gamma \delta}^{\star}(\omega)= & \widetilde{U}_{\gamma \delta}-\frac{(i \hbar)^{2}}{2} \sum_{\substack{\mu \\
\nu \lambda}} \sum_{\xi \theta} \widetilde{V}_{\gamma \mu, \nu \lambda} \int \frac{d \omega_{1}}{2 \pi} \int \frac{d \omega_{2}}{2 \pi} G_{\nu \xi}\left(\omega_{1}\right) G_{\lambda \theta}\left(\omega_{2}\right) \Gamma_{\xi \theta, \delta \epsilon}^{4-\mathrm{pt}}\left(\omega_{1}, \omega_{2} ; \omega, \omega_{1}+\omega_{2}-\omega\right) G_{\epsilon \mu}\left(\omega_{1}+\omega_{2}-\omega\right) \\
& +\frac{(i \hbar)^{4}}{12} \sum_{\substack{\mu \phi \\
\lambda \nu \chi}} \sum_{\substack{\theta \xi \eta \\
\epsilon \sigma}} W_{\mu \gamma \phi, \lambda \nu \chi} \int \frac{d \omega_{1}}{2 \pi} \int \frac{d \omega_{2}}{2 \pi} \int \frac{d \omega_{3}}{2 \pi} \int \frac{d \omega_{4}}{2 \pi} G_{\lambda \theta}\left(\omega_{1}\right) G_{\nu \xi}\left(\omega_{2}\right) G_{\chi \eta}\left(\omega_{3}\right) \\
& \times \Gamma_{\theta \xi \eta, \epsilon \delta \sigma}^{6-\mathrm{pt}}\left(\omega_{1}, \omega_{2}, \omega_{3} ; \omega_{4}, \omega, \omega_{1}+\omega_{2}+\omega_{3}-\omega_{4}-\omega\right) G_{\epsilon \mu}\left(\omega_{4}\right) G_{\sigma \phi}\left(\omega_{1}+\omega_{2}+\omega_{3}-\omega_{4}-\omega\right) .
\end{aligned}
$$



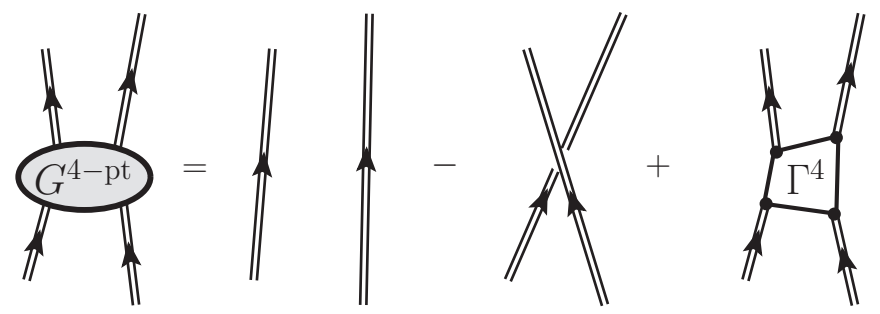

FIG. 7. Exact separation of the four-point Green's function, $G^{4-\mathrm{pt}}$, in terms of noninteracting lines and a vertex function, as given in Eq. (23). The first two terms are the direct and exchange propagation of two noninteracting and fully dressed particles. The last term defines the four-point vertex function, $\Gamma^{4-p t}$, involving the sum of all 1PI diagrams.

The diagrammatic representation of Eq. (25) is shown in Fig. 9. We note that, as an irreducible self-energy, this should include all the connected, 1PI diagrams. These can be regrouped in terms of skeleton and interaction-irreducible contributions, as long as $\Gamma^{4-\mathrm{pt}}$ and $\Gamma^{6-\mathrm{pt}}$ are expressed that way. Note that effective interactions are used here. The interaction-reducible components of $\widetilde{U}, \widetilde{V}$, and $W$ are actually generated by contributions involving partially noninteracting propagators contributions inside $G^{4-\mathrm{pt}}$ and $G^{6-\mathrm{pt}}$. The first two terms in both Eqs. (23) and (24) only contribute to generate effective interactions. Note, however, that the $2 \mathrm{~B}$ effective interaction does receive contributions from both $\Gamma^{4-p t}$ and $\Gamma^{6-p t}$ in the self-consistent procedure.

The first term entering Eq. (25) is the energy-independent contribution to the irreducible self-energy, already found in Eq. (16). This includes the subtraction of the auxiliary field $\hat{U}$, as well as the $1 \mathrm{~B}$ interaction-irreducible contributions due to the $2 \mathrm{~B}$ and $3 \mathrm{BFs}$. Once again, we note that the definition of this term, shown in Fig. 1, involves fully correlated density matrices. Consequently, even though this is a static contribution, it goes beyond the Hartree-Fock approximation. The dispersive part of the self-energy is described by the second and third terms on the right-hand side of Eq. (25). These

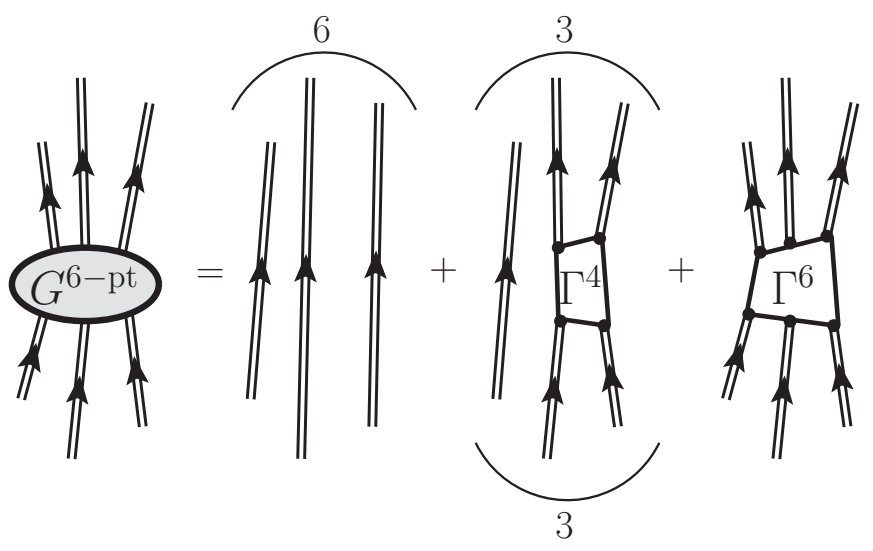

FIG. 8. Exact separation of the six-point Green's function, $G^{6-p t}$, in terms of noninteracting dressed fermion lines and vertex functions, as given in Eq. (24). The first two terms gather noninteracting dressed lines and subgroups of interacting particles that are fully connected to each other. Round brackets with numbers above (below) these diagrams indicate the numbers of permutations of outgoing (incoming) legs needed to generate all possible diagrams. The last term defines the six-point $1 P I$ vertex function $\Gamma^{6-p t}$.

account for all higher-order contributions and incorporate correlations on a $2 \mathrm{~B}$ and $3 \mathrm{~B}$ level associated with the vertex functions $\Gamma^{4-p t}$ and $\Gamma^{6-p t}$, respectively. In Sec. III C below, we will expand these vertices up to second order and show that Eq. (25) actually generates all diagrams derived in Sec. II B.

\section{B. Equation of motion for $G^{4-p t}$ and $\Gamma^{4-p t}$}

We now apply the EOM method to the four-point GF. This will provide insight into approximation schemes that involve correlations at or beyond the $2 \mathrm{~B}$ level. Let us stress that our final aim is to obtain generic nonperturbative approximation schemes in the many-body sector. Taking the time derivative of the first argument in Eq. (3) and following the same procedure as in Sec. III A, we find

$$
\begin{aligned}
G_{\alpha \gamma, \beta \delta}^{4-\mathrm{pt}}\left(\omega_{\alpha}, \omega_{\gamma} ; \omega_{\beta}, \omega_{\delta}\right)= & i \hbar\left[2 \pi \delta\left(\omega_{\alpha}-\omega_{\beta}\right) G_{\alpha \beta}^{(0)}\left(\omega_{\alpha}\right) G_{\gamma \delta}\left(\omega_{\gamma}\right)-2 \pi \delta\left(\omega_{\gamma}-\omega_{\beta}\right) G_{\alpha \delta}^{(0)}\left(\omega_{\alpha}\right) G_{\gamma \beta}\left(\omega_{\gamma}\right)\right] \\
& +\sum_{\mu \lambda} G_{\alpha \mu}^{(0)}\left(\omega_{\alpha}\right) U_{\mu \lambda} G_{\lambda \gamma, \beta \delta}^{4-\mathrm{pt}}\left(\omega_{\alpha}, \omega_{\gamma} ; \omega_{\beta}, \omega_{\delta}\right) \\
& -\frac{1}{2} \sum_{\mu \epsilon} G_{\alpha \mu}^{(0)}\left(\omega_{\alpha}\right) V_{\mu \epsilon, \lambda \theta} \int \frac{d \omega_{1}}{2 \pi} \int \frac{d \omega_{2}}{2 \pi} G_{\lambda \theta \gamma, \beta \epsilon \delta}^{6-\mathrm{pt}}\left(\omega_{1}, \omega_{2}, \omega_{\gamma} ; \omega_{\beta}, \omega_{1}+\omega_{2}-\omega_{\alpha}, \omega_{\delta}\right) \\
& +\frac{1}{12} \sum_{\mu \epsilon \chi} G_{\alpha \mu}^{(0)}\left(\omega_{\alpha}\right) W_{\mu \epsilon \chi, \lambda \theta \eta} \int \frac{d \omega_{1}}{2 \pi} \int \frac{d \omega_{2}}{2 \pi} \int \frac{d \omega_{3}}{2 \pi} \int \frac{d \omega_{4}}{2 \pi} \\
& \times G_{\lambda \theta \eta \eta}^{8-p t} \underset{\lambda \epsilon \in \chi}{2 \pi}\left(\omega_{1}, \omega_{2}, \omega_{3}, \omega_{\gamma} ; \omega_{\beta}, \omega_{4}, \omega_{1}+\omega_{2}+\omega_{3}-\omega_{\alpha}-\omega_{4}, \omega_{\delta}\right),
\end{aligned}
$$

which is analogous to Eq. (22) for the SP propagator. As expected, the EOM connects the two-body (four-point) GFs to other propagators. The 1B propagator term just provides the noninteracting dynamics, with the proper antisymmetrization. The interactions bring in admixtures with the four-point GFs itself, via the one-body potential, but also with the six- and eight-point GFs, via the the 2B and the 3B interactions, respectively. Similarly to what we observed in Eq. (22), the dynamics involve $n-4$ 


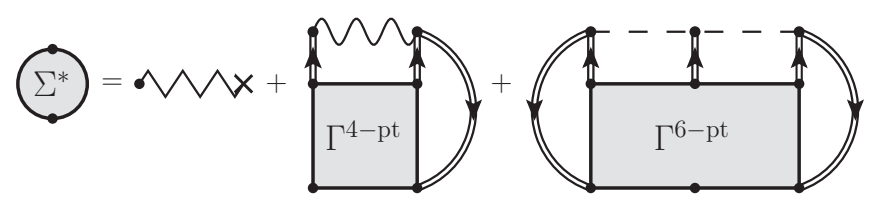

FIG. 9. Diagrammatic representation of the irreducible selfenergy $\Sigma^{\star}$ by means of effective $1 \mathrm{~B}$ and $2 \mathrm{~B}$ potentials and 1PI vertex functions, as given in Eq. (25). The first term is the energyindependent part of $\Sigma^{\star}$ and contains all diagrams depicted in Fig. 1. The second and third terms are dynamical terms consisting of excited configurations generated through $2 \mathrm{~B}$ and $3 \mathrm{BFs}$. This is an exact equation for Hamiltonians including $3 \mathrm{BFs}$ and it is not derived from perturbation theory.

frequency integrals of the $n$-point GFs. The diagrammatic representation of this equation is given in Fig. 10.

To proceed further, we follow the steps of the previous section and of Ref. [60] and split the eight-point GF into free dressed propagators and 1PI vertex functions. This decomposition is shown in Fig. 11. In addition to the alreadydefined vertex functions, one needs 1PI objects with four incoming and outgoing indices. To this end, we introduce the eight-point vertex $\Gamma^{8-\mathrm{pt}}$ in the last term. Note that due care has to be taken of all antisymmetrization possibilities when groups of fermion lines that are not connected by $\Gamma^{8-\mathrm{pt}}$ are considered. The first term, for instance, involves four noninteracting but dressed fermion lines, and there are $4 !=24$ possible combinations. There are $\left(\begin{array}{l}4 \\ 2\end{array}\right)\left(\begin{array}{l}4 \\ 2\end{array}\right) \frac{1}{2}=72$ equivalent terms involving two noninteracting lines and a single $\Gamma^{4-p t}$, as in the second term of Fig. 11. The double $\Gamma^{4-\mathrm{pt}}$ contribution (third term) can be obtained in $6 \times 3=18$ equivalent ways.
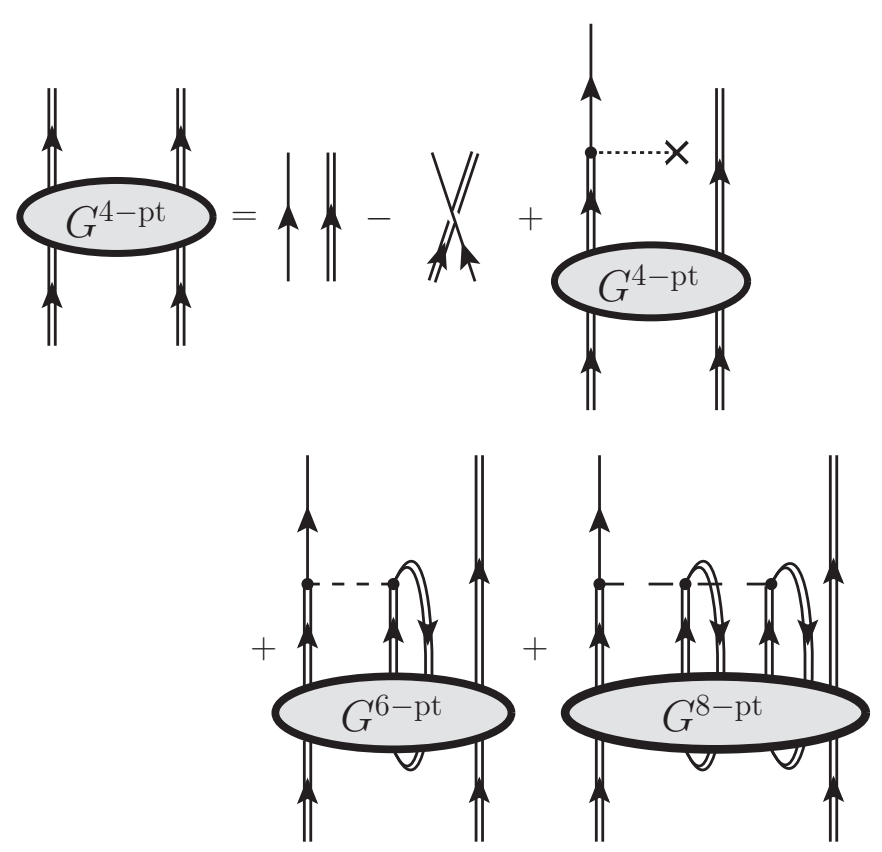

FIG. 10. Diagrammatic representation of the EOM for the fourpoint propagator, $G^{4-p t}$, given in Eq. (26). The last term, involving an eight-point GF, arises due to the presence of $3 \mathrm{~B}$ interactions.
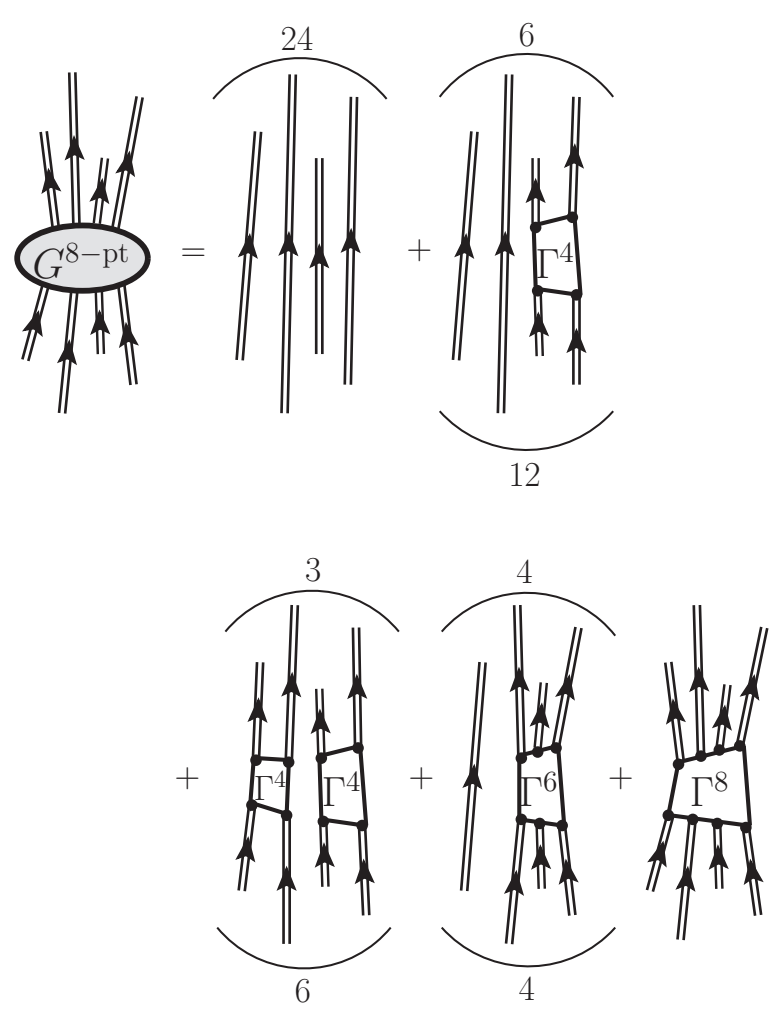

FIG. 11. Exact separation of the eight-point Green's function, $G^{8-\mathrm{pt}}$, in terms of noninteracting lines and vertex functions. The first four terms gather noninteracting dressed lines and subgroups of interacting particles that are fully connected to each other. Round brackets with numbers above (below) these diagrams indicate the numbers of permutations of outgoing (incoming) legs needed to generate all possible diagrams. The last term defines the eight-point 1PI vertex function $\Gamma^{8-\mathrm{pt}}$.

With this decomposition at hand, one can now proceed and find an equation for the four-point vertex function, $\Gamma^{4-p t}$. Inserting the exact decompositions of the four-, six- and eightpoint GFs, given, respectively, by Figs. 7, 8, and 11, into the EOM [Eq. (26)], one obtains an equation with $\Gamma^{4-p t}$ on both sides. The diagrammatic representation of this self-consistent equation is shown in Fig. 12.

A few comments are in order at this point. The left-hand side of Eq. (26) in principle contains two dressed and noninteracting propagators, as shown in the first two terms of Fig. 7. In the right-hand side, however, one of the $1 \mathrm{~B}$ propagators is not dressed. When expanding the GFs in Eq. (26) in terms of the $\Gamma^{2 n-p t}$ vertex functions, the remaining contributions to the Dyson equation arise automatically (Fig. 6). The free unperturbed line, therefore, becomes dressed. As a consequence, the pair of dressed noninteracting propagators cancel out exactly on both sides of Eq. (26). This dressing procedure of the $G^{(0)}$ propagator happens only partially in the last three terms of the equation and was disregarded in our derivation. In this sense, Fig. 12 should be taken as an approximation to the exact EOM for $G^{4-\mathrm{pt}}$.

Equation (26) links 1B, 2B, 3B, and 4B propagators. Correspondingly, Fig. 12 involves higher-order vertex functions, 


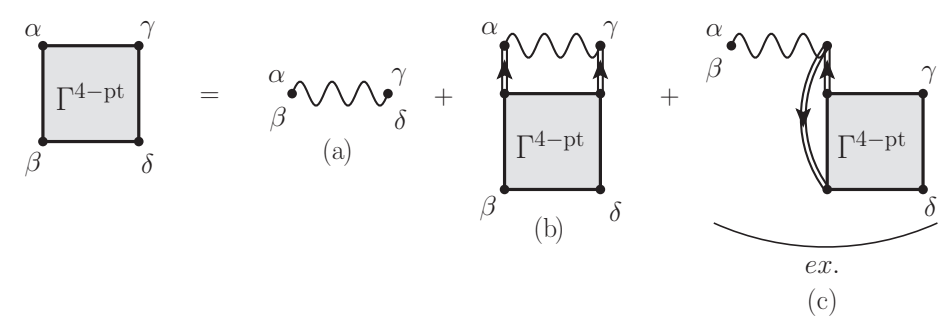

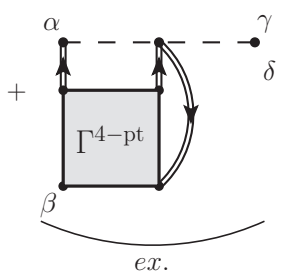

(d)

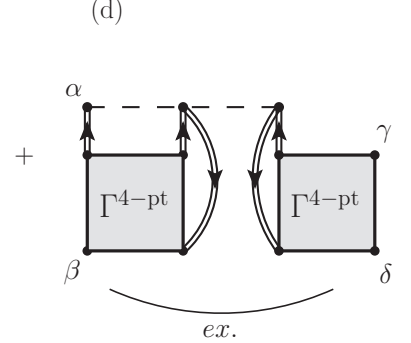

(i)

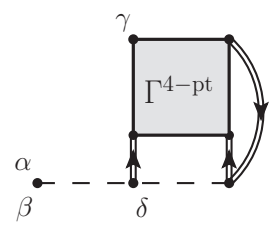

(e)

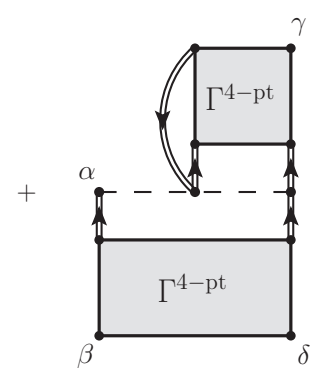

(j)

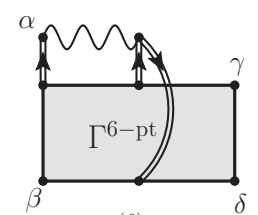

(f)

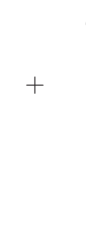

$\beta$

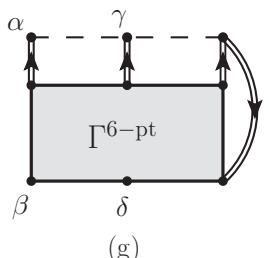

(g)

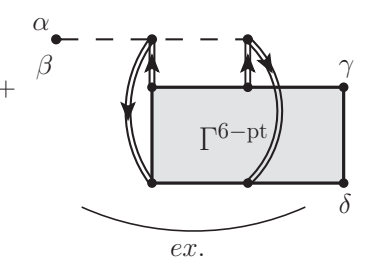

(h)

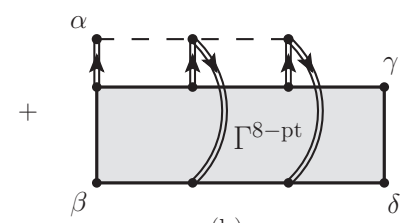

$(\mathrm{k})$

FIG. 12. Self-consistent expression for the $\Gamma^{4-\mathrm{pt}}$ vertex function derived from the EOM for $G^{4-\mathrm{pt}}$. The round brackets underneath some of the diagrams indicate that the term obtained by exchanging the $\left\{\beta \omega_{\beta}\right\}$ and $\left\{\delta \omega_{\delta}\right\}$ arguments must also be included. Diagrams (a), (b), (c), and (f) are the only ones present for 2B Hamiltonians, although (f) also contains some intrinsic $3 \mathrm{BF}$ contributions such as the $\left\{\alpha \omega_{\alpha}\right\} \leftrightarrow\left\{\gamma \omega_{\gamma}\right\}$ exchange of (e). All other diagrams arise from the inclusion of 3B interactions. Diagram (b) is responsible for generating the ladder summation, the direct part of (c) generates the series of antisymmetrized rings, and the three sets together [(b), (c), and the exchange of (c)] would give rise to a Parquet-type resummation.

such as $\Gamma^{6-\mathrm{pt}}$ and $\Gamma^{8-\mathrm{pt}}$, which are in principle coupled, through their own EOMs, to more complex GFs. The hierarchy of these equations has to be necessarily truncated. In Ref. [60], truncation schemes were explored by neglecting the $\Gamma^{6-\mathrm{pt}}$ vertex function at the level of Fig. $12\left(\Gamma^{8-p t}\right.$ did not appear in the 2BF-only case). This level of truncation is already sufficient to retain physically relevant subsets of diagrams, such as ladders and rings. Let us note, in particular, that the summation of these infinite series leads to nonperturbative many-body schemes. For completeness, we show in Fig. 12 all contributions coming also from the $\Gamma^{6-\mathrm{pt}}$ and $\Gamma^{8-\mathrm{pt}}$ vertices, many of them arising from 3BFs.

We have ordered the diagrams in Fig. 12 in terms of increasing contributions from $3 \mathrm{BFs}$ and in the order of perturbation theory at which they start contributing to $\Gamma^{4-\mathrm{pt}}$. Intuitively, we expect that this should order them in decreasing importance. Diagrams Figs. 12(a)-12(c) and 12(f) are those that are also present in the 2BF-only case. Diagram Fig. 12(f), however, is of a mixed nature: It can contribute only at third order with effective $2 \mathrm{BFs}$, but does contain interactionirreducible $3 \mathrm{BF}$ contributions at second order that are similar to diagrams Figs. 12(d) and 12(e). Diagrams Figs. 12(d)-12(h) all contribute to $\Gamma^{4-\mathrm{pt}}$ at second order, although the first three require a combination of a $\widetilde{V}$ and a $W$ term. The remaining diagrams in this group, Figs. 12(g) and 12(h), require two 3B interactions at second order and are expected to be subleading. Note that Fig. 12(d) is antisymmetric in $\alpha$ and $\gamma$, but it must also be antisymmetrized with respect to $\beta$ and $\delta$. Its conjugate contribution, Fig. 12(e), should not be further antisymmetrized in $\alpha$ and $\gamma$, because such exchange term is already included in Fig. 12(f). All the remaining terms, Figs. 12(i)-12(k), only contribute from the third order on.

The simplest truncation schemes to $\Gamma^{4-p t}$ come from considering the first three terms of Fig. 12, which involve effective 2BFs only. In the pure 2B case, these have already been discussed in the literature [60]. Retaining diagrams Figs. 12(a) and 12(b) leads to the ladder resummation used in recent studies of infinite nucleonic matter [21,27]:

$$
\begin{aligned}
& \Gamma_{\alpha \gamma, \beta \delta}^{4_{\text {ladd }}}\left(\omega_{\alpha}, \omega_{\gamma} ; \omega_{\beta}, \omega_{\alpha}+\omega_{\gamma}-\omega_{\beta}\right)
\end{aligned}
$$

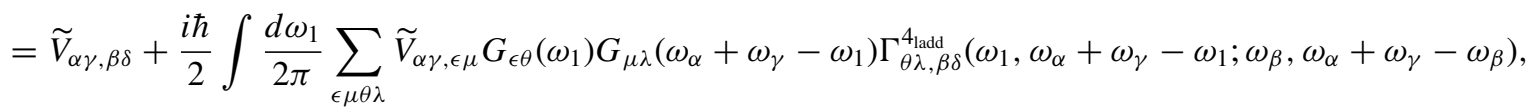


where we have explicitly used the fact that $\Gamma^{2 n-p t}$ is only defined when incoming and outgoing energies are conserved. Likewise, diagrams Fig. 12(a) and the direct contribution of Fig. 12(c) generate a series of ring diagrams which correspond to the antisymmetrized version of the random phase approximation (RPA):

$$
\begin{aligned}
& \Gamma_{\alpha \gamma, \beta \delta}^{4_{\text {ring }}}\left(\omega_{\alpha}, \omega_{\gamma} ; \omega_{\beta}, \omega_{\alpha}+\omega_{\gamma}-\omega_{\beta}\right) \\
& \quad=\widetilde{V}_{\alpha \gamma, \beta \delta}-i \hbar \int \frac{d \omega_{1}}{2 \pi} \sum_{\epsilon \mu \theta \lambda} \widetilde{V}_{\alpha \epsilon, \beta \mu} G_{\mu \lambda}\left(\omega_{1}\right) G_{\theta \epsilon}\left(\omega_{1}-\omega_{\alpha}+\omega_{\beta}\right) \Gamma_{\lambda \gamma, \theta \delta}^{4_{\text {ring }}}\left(\omega_{1}, \omega_{\gamma} ; \omega_{1}-\omega_{\alpha}+\omega_{\beta}, \omega_{\alpha}+\omega_{\gamma}-\omega_{\beta}\right) .
\end{aligned}
$$

Adding up the first three contributions together, Figs. 12(a)-12(c), and including the exchange, will generate a Parquet-type of resummation, with ladders and rings embedded into each other:

$$
\begin{aligned}
& \Gamma_{\alpha \gamma, \beta \delta}^{4_{\text {Parquet }}}\left(\omega_{\alpha}, \omega_{\gamma} ; \omega_{\beta}, \omega_{\alpha}+\omega_{\gamma}-\omega_{\beta}\right) \\
& =\widetilde{V}_{\alpha \gamma, \beta \delta}+i \hbar \int \frac{d \omega_{1}}{2 \pi} \sum_{\epsilon \mu \theta \lambda}\left[\frac{1}{2} \widetilde{V}_{\alpha \gamma, \epsilon \mu} G_{\epsilon \theta}\left(\omega_{1}\right) G_{\mu \lambda}\left(\omega_{\alpha}+\omega_{\gamma}-\omega_{1}\right) \Gamma_{\theta \lambda, \beta \delta}^{4 \text { Parquet }}\left(\omega_{1}, \omega_{\alpha}+\omega_{\gamma}-\omega_{1} ; \omega_{\beta}, \omega_{\alpha}+\omega_{\gamma}-\omega_{\beta}\right)\right. \\
& \quad-\widetilde{V}_{\alpha \epsilon, \beta \mu} G_{\mu \lambda}\left(\omega_{1}\right) G_{\theta \epsilon}\left(\omega_{1}-\omega_{\alpha}+\omega_{\beta}\right) \Gamma_{\lambda \gamma, \theta \delta}^{4 \text { Paraut }}\left(\omega_{1}, \omega_{\gamma} ; \omega_{1}-\omega_{\alpha}+\omega_{\beta}, \omega_{\alpha}+\omega_{\gamma}-\omega_{\beta}\right) \\
& \left.\quad+\widetilde{V}_{\alpha \epsilon, \delta \mu} G_{\mu \lambda}\left(\omega_{1}\right) G_{\theta \epsilon}\left(\omega_{1}+\omega_{\gamma}-\omega_{\beta}\right) \Gamma_{\lambda \gamma, \theta \beta}^{4 \text { Parquet }}\left(\omega_{1}, \omega_{\gamma} ; \omega_{1}+\omega_{\gamma}-\omega_{\beta}, \omega_{\beta}\right)\right] .
\end{aligned}
$$

Equations (27) and (28) can be solved in a more or less simple fashion because the corresponding vertex functions effectively depend on only one frequency ( $\Omega=\omega_{\alpha}+\omega_{\gamma}$ and $\Omega=\omega_{\alpha}-\omega_{\beta}$, respectively). Hence these two resummation schemes have been traditionally used to study extended systems $[7,8,14]$. The simultaneous resummation of both rings and ladders within the self-energy is possible for finite systems, and it is routinely used in both quantum chemistry and nuclear physics $[11,35,61,62]$. The Parquet summation, as shown in Eq. (29), does require all three independent frequencies and it is difficult to implement numerically. Specific approximations to rewrite these in terms of two-time vertex functions have been recently attempted [63], but further developments are still required.

The next approximation to $\Gamma^{4-p t}$ would involve diagrams Figs. 12(d) and 12(e), and the exchange part included in Fig. 12(f). All these should be added together to preserve the antisymmetry and conjugate properties of the vertex function. The resulting contributions still depend on all three frequencies and cannot be simply embedded in all-order summations such as the ladder, Eq. (27), or the ring, Eq. (28), approximations. However, these diagrams could be used to obtain corrections, at first order in the interaction-irreducible $\hat{W}$, to the previously calculated four-point vertices. The explicit expression for these terms is

$$
\begin{aligned}
\Delta \Gamma_{\alpha \gamma, \beta \delta}^{4_{d+e+e^{\prime}}}\left(\omega_{\alpha}, \omega_{\gamma} ; \omega_{\beta}, \omega_{\alpha}+\omega_{\gamma}-\omega_{\beta}\right) \\
=\frac{(i \hbar)^{2}}{2} \int \frac{d \omega_{1}}{2 \pi} \int \frac{d \omega_{2}}{2 \pi} \sum_{\substack{\epsilon \mu \xi \\
\theta \lambda \nu}}\left[-W_{\alpha \nu \gamma, \epsilon \mu \delta} G_{\epsilon \theta}\left(\omega_{1}\right) G_{\mu \lambda}\left(\omega_{2}\right) \Gamma_{\theta \lambda, \beta \xi}\left(\omega_{1}, \omega_{2} ; \omega_{\beta}, \omega_{1}+\omega_{2}-\omega_{\beta}\right) G_{\xi \nu}\left(\omega_{1}+\omega_{2}-\omega_{\beta}\right)\right. \\
\quad+W_{\alpha \nu \gamma, \epsilon \mu \beta} G_{\epsilon \theta}\left(\omega_{1}\right) G_{\mu \lambda}\left(\omega_{2}\right) \Gamma_{\theta \lambda, \delta \xi}\left(\omega_{1}, \omega_{2} ; \omega_{\alpha}+\omega_{\gamma}-\omega_{\beta}, \omega_{1}+\omega_{2}-\omega_{\alpha}-\omega_{\gamma}+\omega_{\beta}\right) G_{\xi \nu}\left(\omega_{1}+\omega_{2}-\omega_{\alpha}-\omega_{\gamma}+\omega_{\beta}\right) \\
\quad-\Gamma_{\gamma \nu, \epsilon \mu}\left(\omega_{\gamma}, \omega_{1}+\omega_{2}-\omega_{\gamma} ; \omega_{1}, \omega_{2}\right) G_{\epsilon \theta}\left(\omega_{1}\right) G_{\mu \lambda}\left(\omega_{2}\right) W_{\alpha \theta \lambda, \beta \delta \xi} G_{\xi \nu}\left(\omega_{1}+\omega_{2}-\omega_{\gamma}\right) \\
\left.\quad+\Gamma_{\alpha \nu, \epsilon \mu}\left(\omega_{\alpha}, \omega_{1}+\omega_{2}-\omega_{\alpha} ; \omega_{1}, \omega_{2}\right) G_{\epsilon \theta}\left(\omega_{1}\right) G_{\mu \lambda}\left(\omega_{2}\right) W_{\gamma \theta \lambda, \beta \delta \xi} G_{\xi \nu}\left(\omega_{1}+\omega_{2}-\omega_{\alpha}\right)\right] .
\end{aligned}
$$

Equation (30) has some very attractive features. First, it should provide the dominant contribution beyond those associated with the effective 2B interaction $\widetilde{V}$. Perhaps more importantly, this contribution can be easily calculated in terms of one of the two-time vertex functions, $\Gamma^{4_{\text {ladd }}}$ and $\Gamma^{4_{\text {ring }}}$. This could then be inserted in Eq. (23) to generate corrections of expectation values of $2 \mathrm{~B}$ operators stemming from purely irreducible $3 \mathrm{~B}$ contributions. A similar correction for the irreducible self-energy is also discussed in the next section.

Once a truncation scheme is chosen at the level of the vertex functions, one can immediately derive a diagrammatic approximation for the self-energy [3]. Conserving approximations can plausibly be derived from some of these truncation schemes [56]. A general derivation of $\Phi$ derivability with
3BF should be possible, but goes beyond the scope of this work.

\section{Contributions to the irreducible self-energy}

In this subsection, we demonstrate the correspondence between the techniques derived in Sec. II and the EOM method. In particular, we want to show how the perturbative expansion of Eq. (7) leads to the self-energy obtained with the EOM expression [Eq. (25)]. We will do this by expanding the self-energy up to third order and showing the equivalence of both approaches at this order. To this end, we need to expand the vertex functions in terms of the effective Hamiltonian $\widetilde{H}_{1}$. The lowest order terms entering $\Gamma^{4-\mathrm{pt}}$ can be easily 

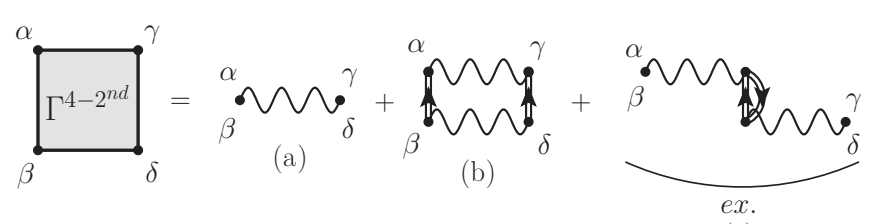

(c)

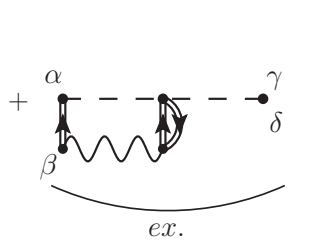

(d)

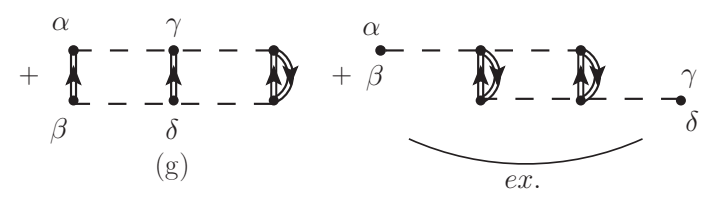

(h)

FIG. 13. Skeleton and interaction-irreducible diagrams contributing to the $\Gamma^{4-\mathrm{pt}}$ vertex function up to second order. The round brackets above (below) some diagrams indicate that the exchange diagram between the $\left\{\alpha \omega_{\alpha}\right\}$ and $\left\{\gamma \omega_{\gamma}\right\}\left(\left\{\beta \omega_{\beta}\right\}\right.$ and $\left.\left\{\delta \omega_{\delta}\right\}\right)$ arguments must also be included.

read from Fig. 12. We show these second-order, skeleton and interaction-irreducible diagrams in Fig. 13. Only the first three terms would contribute for a $2 \mathrm{BF}$. There are two terms involving mixed $2 \mathrm{BFs}$ and $3 \mathrm{BF}$, whereas the final two contributions come from two independent $3 \mathrm{BFs}$. Note that, to get the third-order expressions of the self-energy, we expand the vertex functions to second order, i.e., one order less.

Analogously, we display the expansion up to second order of $\Gamma^{6-p t}$ in Fig. 14. Most contributions to this vertex function contain 3BFs. The lowest order term, for instance, is given by the $3 \mathrm{~B}$ interaction itself. Note, however, that second-order terms formed of $2 \mathrm{~B}$ effective interactions are possible, such as the second term on the right-hand side of Fig. 14. These will eventually be connected with a $3 \mathrm{BF}$ to give a mixed self-energy contribution [see Eq. (25) and Fig. 9].

If one includes the diagrams in Figs. 13 and 14 into the irreducible self-energy $\Sigma^{\star}$ of Fig. 9, all the diagrams discussed in Eq. (16), Figs. 3 and 5 of Sec. II B are recovered. This does prove, at least up to third order, the correspondence between the perturbative expansion approach and the EOM method for the GFs. Proceeding in this manner to higher orders, one should obtain equivalent diagrams all the way through.

It is important to note that diagrams representing conjugate contributions to $\Sigma^{\star}$ are generated by different, not necessarily conjugate, terms of $\Gamma^{4-\mathrm{pt}}$ and $\Gamma^{6-\mathrm{pt}}$. For instance, diagram Fig. 5(d) is the result of the term 13(e+f) and its exchange, on the right-hand side of Fig. 13. Its conjugate self-energy diagram, Fig. 5(f), however, is generated by the second contribution to $\Gamma^{6-p t}$, Fig. 14(b). This term is also related to diagram Fig. 5(g). More specifically, the term 14(b) has nine cyclic permutations of its indices, of which six contribute to diagram Fig. 5(f) and three to diagram Fig. 5(g). On the other

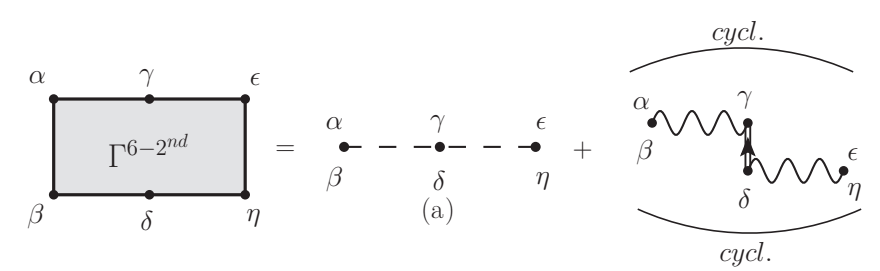

(b)
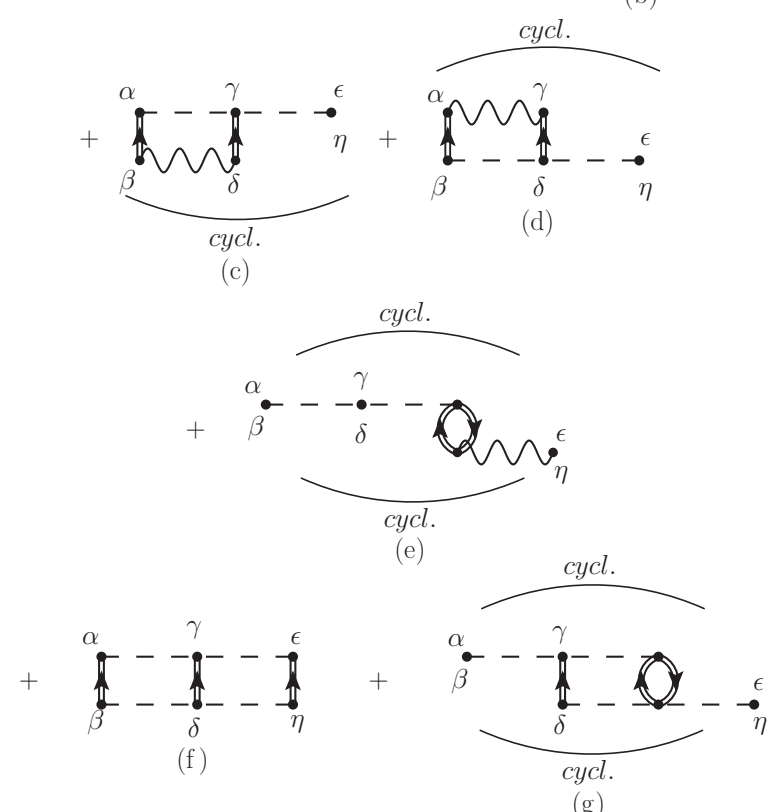

FIG. 14. The same as Fig. 13 for the $\Gamma^{6-\mathrm{pt}}$ vertex function. The round brackets above (below) some diagrams indicate that cyclic permutations of the $\left\{\alpha \omega_{\alpha}\right\},\left\{\gamma \omega_{\gamma}\right\}$ and $\left\{\epsilon \omega_{\epsilon}\right\}\left(\left\{\beta \omega_{\beta}\right\},\left\{\delta \omega_{\delta}\right\}\right.$ and $\left.\left\{\eta \omega_{\eta}\right\}\right)$ arguments must also be included.

hand, the conjugate of Fig. 5(g) is diagram Fig. 5(e), which is entirely due to the exchange contribution of the Fig. 13(d) term in $\Gamma^{4-p t}$. The direct contribution of this same term leads to diagram Fig. 5(c), which is already self-conjugate.

More importantly, however, nonperturbative self-energy expansions can be obtained by means of other hierarchy truncations at the level of $\Gamma^{4-\mathrm{pt}}$ and $\Gamma^{6-\mathrm{pt}}$. Translating these into self-energy expansions is then just an issue of introducing them in Eq. (25). According to the approximation chosen for the vertex functions appearing in Fig. 9, we will be summing specific sets of diagrams when solving the Dyson equation, Eq. (8). However, from the above discussion it should be clear that extra care must be taken to guarantee that the truncations lead to physically coherent results. In particular, it is not always possible to naively neglect $\Gamma^{6-p t}$. The last two terms of the self-energy equation, Eq. (25), generate conjugate contributions. Hence, neglecting one term or the other will spoil the analytic properties of the self-energy which require a Hermitian real part and an anti-Hermitian imaginary part. In the examples discussed above, diagrams Figs. 5(f) and 5(g) would be missing if $\Gamma^{6-\mathrm{pt}}$ had not been considered.

When no irreducible 3B interaction terms are present in the hierarchy truncation, only the $\Gamma^{4-p t}$ term contributes to Eq. (25). The ladder and the ring truncations, shown in 


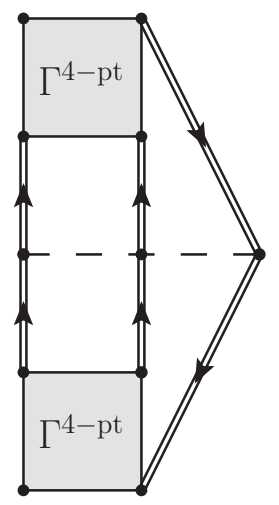

FIG. 15. Diagrammatic representation of the self-energy correction $\Delta \Sigma^{\star} \Gamma \omega \Gamma$ given in Eq. (31).

Eqs. (27) and (28) generate their own conjugate diagrams and can be used on their own to obtain physical approximations to the self-energy. However, this need not be true in general. A counterexample is actually provided by the truncation of Eq. (30) which, if inserted in Eq. (25) without the corresponding contributions to $\Gamma^{6-\mathrm{pt}}$, cannot generate a correct self-energy. Because of its diagrammatic content, Eq. (30) can only be used as a correction to $\Gamma^{4-p t}$.

As far as 3B interaction-irreducible diagrams are concerned, the most important contribution should be that associated with Fig. 5(c) as discussed in Sec. II B. Further contributions with similar structures are also expected to contribute to the correlation dynamics. To include such terms, one can go beyond third order by replacing the effective $2 \mathrm{BFs}$ at the upper and lower ends by ladder or ring summations. Note that this is precisely the structure that arises from the hierarchy truncation associated with Eq. (30). This would lead to a generalized contribution, whose diagrammatic content is summarized by Fig. 15. The corresponding expression for the self-energy would read

$$
\begin{aligned}
\Delta \Sigma_{\alpha \beta}^{\star_{\Gamma} W \Gamma}(\omega)= & -\frac{(i \hbar)^{4}}{4} \int \frac{d \omega_{1}}{2 \pi} \cdots \int \frac{d \omega_{4}}{2 \pi} \sum_{\substack{\gamma \delta \nu \\
\sigma \tau \chi}} \sum_{\substack{\mu \in \lambda \\
\xi \eta \theta}} \\
& \times \Gamma_{\alpha \gamma, \delta \nu}^{4-\mathrm{pt}}\left(\omega, \omega_{1}+\omega_{2}-\omega ; \omega_{1}, \omega_{2}\right) G_{\delta \mu}\left(\omega_{1}\right) \\
& \times G_{\nu \epsilon}\left(\omega_{2}\right) G_{\theta \gamma}\left(\omega_{1}+\omega_{2}-\omega\right) W_{\mu \epsilon \lambda, \xi \eta \theta} G_{\xi \sigma}\left(\omega_{3}\right) \\
& \times G_{\eta \tau}\left(\omega_{4}\right) G_{\chi \lambda}\left(\omega_{3}+\omega_{4}-\omega\right) \\
& \times \Gamma_{\sigma \tau, \beta \chi}^{4-\mathrm{pt}}\left(\omega_{3}, \omega_{4} ; \omega, \omega_{3}+\omega_{4}-\omega\right) .
\end{aligned}
$$

To quantify the importance of these terms, they would need to be included in the self-consistent procedure. Moreover, these corrections should also be considered when computing the total energy, as we will see next.

To conclude this section, we would like to stress the fact that extensions to include $3 \mathrm{BFs}$ beyond effective $2 \mathrm{~B}$ interactions, like $\widetilde{V}$, are a completely virgin territory. To our knowledge, these have not been evaluated for nuclear systems (or any other system, for that matter) with diagrammatic formalisms. Truncation schemes, like those proposed here, should provide insight on in-medium 3B correlations. The advantage that the SCGF formalism provides is the access to nonperturbative, conserving approximations that contain pure 3B dynamics without the need for ad hoc assumptions.

\section{GROUND-STATE ENERGY}

SCGF calculations aim at providing reliable calculations for the SP propagator of correlated systems via diagrammatic techniques. Traditionally, there have been two motivations to do this. On the one hand, the SP propagator provides access to all $1 \mathrm{~B}$ operators and hence is a useful tool to characterize a wide range of the system's properties. On the other hand, the ground-state energy is a critically important $2 \mathrm{~B}$ observable that can be obtained from the 1B GF itself. This is a crucial result, that arises from the GMK sum rule [39,40]. The sum rule is valid both at zero and at finite temperature, where the $1 \mathrm{~B}$ propagator also provides access to the energy and, at least approximately, to all other thermodynamical properties [64]. In this section, we investigate the modifications of the GMK sum rule when $3 \mathrm{BF}$ are included in the Hamiltonian.

Not all the information content from the propagator is needed to obtain the ground-state energy. The hole part, which includes details about the transition amplitude for the removal of a particle from the many-body system, is enough for these purposes. One therefore defines the diagonal part of the hole spectral function:

$$
\begin{aligned}
\mathcal{S}_{\alpha}^{h}(\omega) & =\frac{1}{\pi} \operatorname{Im} G_{\alpha \alpha}(\omega) \\
& =\sum_{n}\left|\left\langle\Psi_{n}^{N-1}\left|a_{\alpha}\right| \Psi_{0}^{N}\right\rangle\right|^{2} \delta\left[\hbar \omega-\left(E_{0}^{N}-E_{n}^{N-1}\right)\right],
\end{aligned}
$$

for energies below the Fermi energy, $\hbar \omega<\varepsilon_{F}^{-}=E_{0}^{N}-E_{0}^{N-1}$. The $n^{\text {th }}$ excited state of the $N-1$ particle system is described by the many-body wave function $\left|\Psi_{n}^{N-1}\right\rangle$ and has a total energy $E_{n}^{N-1}$. The transition amplitude between the $N$ and the $N-1$ body systems is closely related to the definition of the theoretical spectroscopic factor [14] and also provides information on removal strength distributions. The complete spectral function represents a direct link between theory and experiment, as well as determining energy centroids [65].

To obtain the GMK sum rule, one starts by considering the first moment of the hole spectral function:

$$
I_{\alpha}=\int_{-\infty}^{\epsilon_{F}^{-}} d \omega \omega \mathcal{S}_{\alpha}^{h}(\omega) .
$$

From the spectral representation above, it is easy to see that this sum rule is also the expectation value over the many-body state of the commutator:

$$
I_{\alpha}=\left\langle\Psi_{0}^{N}\left|a_{\alpha}^{\dagger}\left[a_{\alpha}, \hat{H}\right]\right| \Psi_{0}^{N}\right\rangle .
$$

Using the Hamiltonian in Eq. (1), one can evaluate the commutator to find

$$
\begin{aligned}
I_{\alpha}= & \left\langle\Psi_{0}^{N}\right| \sum_{\beta} T_{\alpha \beta} a_{\alpha}^{\dagger} a_{\beta}^{\dagger}+\frac{1}{2} \sum_{\gamma \beta \delta} V_{\alpha \gamma, \beta \delta} a_{\alpha}^{\dagger} a_{\gamma}^{\dagger} a_{\delta} a_{\beta} \\
& +\frac{1}{12} \sum_{\gamma \in \beta \delta \eta} W_{\alpha \gamma \epsilon, \beta \delta \eta} a_{\alpha}^{\dagger} a_{\gamma}^{\dagger} a_{\epsilon}^{\dagger} a_{\eta} a_{\delta} a_{\beta}\left|\Psi_{0}^{N}\right\rangle .
\end{aligned}
$$


Note that, in general, $T$ represents the 1B part of the Hamiltonian which, in addition to the kinetic energy, might also contain the 1B potential. Summing over all the external SP states, $\alpha$, one finds

$$
\sum_{\alpha} I_{\alpha}=\left\langle\Psi_{0}^{N}|\hat{T}+2 \hat{V}+3 \hat{W}| \Psi_{0}^{N}\right\rangle
$$

In other words, the sum over SP states of the first moment of the spectral function yields a particular linear combination of the contributions of the $1 \mathrm{~B}, 2 \mathrm{~B}$, and $3 \mathrm{~B}$ potentials to the ground-state energy,

$$
E_{0}^{N}=\left\langle\Psi_{0}^{N}|\hat{H}| \Psi_{0}^{N}\right\rangle=\left\langle\Psi_{0}^{N}|\hat{T}+\hat{V}+\hat{W}| \Psi_{0}^{N}\right\rangle .
$$

Because $\hat{T}$ is a $1 \mathrm{~B}$ operator, one can actually compute its expectation value from the SP propagator itself:

$$
\left\langle\Psi_{0}^{N}|\hat{T}| \Psi_{0}^{N}\right\rangle=\frac{1}{\pi} \int_{-\infty}^{\epsilon_{F}^{-}} d \omega \sum_{\alpha \beta} T_{\alpha \beta} \operatorname{Im} G_{\beta \alpha}(\omega) .
$$

The energy integral on the right-hand side yields the 1B density matrix element, Eq. (13):

$$
\rho_{\beta \alpha}^{1 B}=\frac{1}{\pi} \int_{-\infty}^{\epsilon_{F}^{-}} d \omega \operatorname{Im} G_{\beta \alpha}(\omega),
$$

which can be used to simplify the previous expression. For the $2 \mathrm{~B}$ case, this is enough to provide an independent constraint and hence allows for the calculation of the total energy. The ground-state energy can then be computed from the $1 \mathrm{~B}$ propagator alone.

When 3BFs are present, however, one needs a third independent linear combination of $\langle\hat{T}\rangle,\langle\hat{V}\rangle$, and $\langle\hat{W}\rangle$. Knowledge of the $1 \mathrm{~B}$ propagator is therefore not enough to compute the total energy, because either the $2 \mathrm{~B}$ or the $3 \mathrm{~B}$ propagators are needed to compute $\langle\hat{V}\rangle$ or $\langle\hat{W}\rangle$ exactly. Depending on which of the two operators is chosen, one is left with different expressions for the energy of the ground state. This freedom in choice could in principle be exploited to test the validity of different approximations. In practical applications, however, one should choose the combination that provides minimum uncertainty.

Let us start by considering the case where the $3 \mathrm{~B}$ operator is eliminated. Adding $2\langle\hat{T}\rangle$ and $\langle\hat{V}\rangle$ to the sum rule, Eq. (36), one finds the following exact expression for the total ground-state energy:

$$
\begin{aligned}
E_{0}^{N}= & \frac{1}{3 \pi} \int_{-\infty}^{\epsilon_{F}^{-}} d \omega \sum_{\alpha \beta}\left(2 T_{\alpha \beta}+\omega \delta_{\alpha \beta}\right) \operatorname{Im} G_{\beta \alpha}(\omega) \\
& +\frac{1}{3}\left\langle\Psi_{0}^{N}|\widehat{V}| \Psi_{0}^{N}\right\rangle .
\end{aligned}
$$

The calculation of this expression requires the hole part of the $1 \mathrm{~B}$ propagator and the two-hole part of the $2 \mathrm{~B}$ propagator, which would appear in the second term. We note that this expression is somewhat equivalent to the original GMK, in that the ground-state energy is computed from $1 \mathrm{~B}$ and $2 \mathrm{~B}$ operators, even though the Hamiltonian itself is a $3 \mathrm{~B}$ operator. This might prove advantageous in calculations where the $2 \mathrm{~B}$ propagator is computed explicitly.
Alternatively, one can eliminate the $2 \mathrm{~B}$ contribution from the GMK sum rule by adding $\langle\hat{T}\rangle$ and subtracting $\langle\hat{W}\rangle$ to the sum rule, Eq. (36). This leads to the following expression:

$$
\begin{aligned}
E_{0}^{N}= & \frac{1}{2 \pi} \int_{-\infty}^{\epsilon_{F}^{-}} d \omega \sum_{\alpha \beta}\left(T_{\alpha \beta}+\omega \delta_{\alpha \beta}\right) \operatorname{Im} G_{\beta \alpha}(\omega) \\
& -\frac{1}{2}\left\langle\Psi_{0}^{N}|\hat{W}| \Psi_{0}^{N}\right\rangle .
\end{aligned}
$$

The first term in this expression is formally the same as that obtained in the case where only $2 \mathrm{BFs}$ are present in the Hamiltonian. In that sense, the second term can be thought of as a correction to the total energy associated with the 3BF. Note, however, that the $3 \mathrm{BF}$ does influence the $1 \mathrm{~B}$ propagator on the first term and hence the correction should only be applied at the very end of the self-consistent procedure.

Equations (40) and (41) are both exact. Which of the two is employed in actual calculations will mostly depend on the accuracy associated with the evaluation of the expectation values, $\langle\hat{V}\rangle$ and $\langle\hat{W}\rangle$. If the $2 \mathrm{~B}$ interaction is dominant with respect to the $3 \mathrm{BF}$, for instance, the former will be a large contribution. Small errors in the calculation of the $2 \mathrm{~B}$ propagator could eventually yield artificially large corrections in the ground-state energy. In nuclear physics, the $3 \mathrm{BF}$ expectation value is expected to provide a smaller contribution than the $2 \mathrm{BF}[22,46]$. Consequently, approximations in Eq. (41) should lead to smaller absolute errors. This was the approach that we recently followed in both finite nuclei and infinite nuclear matter [27,35]. In finite nuclei, evaluating $\langle\hat{W}\rangle$ at first order in terms of dressed propagators leads to satisfactory results. However, accuracy is lost if free propagators, $G^{(0)}$ are used instead. Equation (40) may eventually be useful in calculations of infinite matter, in which the $\Gamma^{4-\mathrm{pt}}$ is calculated nonperturbatively.

This first-order approximation with undressed propagators is traditionally used in nuclear structure. In this context, three-body forces have been often discussed in the HartreeFock approximation with Skyrme or Gogny functionals [1,66]. Zero-range forces have also been employed in $a b$ initio-type calculations [67]. It is perhaps instructive to point out at this stage that the previous formulas apply to this case as well. In particular, the Hartree-Fock approximation with 3BF can be alternatively derived from the variational principle, under the assumption that the many-body state is described by a Slater determinant, $\left|\Phi_{0}^{N}\right\rangle$. Diagonalizing an effective 1B hamiltonian leads to a series of Hartree-Fock orbitals with single-particle energies $\varepsilon_{\alpha}$. The total energy under a $2 \mathrm{~B}$ Hamiltonian is not the sum of these energies, but rather requires a correction to avoid double counting [1]. Similarly, in the 3B case, the energy is computed as follows:

$$
E_{0}^{\mathrm{HF}}=\sum_{\alpha} \varepsilon_{\alpha}-\langle\hat{V}\rangle_{\mathrm{HF}}-2\langle\hat{W}\rangle_{\mathrm{HF}}
$$

This result is straightforwardly derived by noticing that, in the Hartree-Fock approximation, the sum rule, Eq. (36), reduces to the first term. Within this approximation, the expectation values can be directly computed from the uncorrelated $1 \mathrm{~B}$ 
density matrix:

$$
\begin{aligned}
\langle\hat{V}\rangle_{\mathrm{HF}} & =\frac{1}{2} \sum_{\substack{\alpha \gamma \\
\beta \delta}} V_{\alpha \gamma, \beta \delta} \rho_{\beta \alpha}^{\mathrm{HF}} \rho_{\delta \gamma}^{\mathrm{HF}}, \\
\langle\hat{W}\rangle_{\mathrm{HF}} & =\frac{1}{6} \sum_{\substack{\alpha \gamma \epsilon \\
\beta \delta \eta}} W_{\alpha \gamma \epsilon, \beta \delta \eta} \rho_{\beta \alpha}^{\mathrm{HF}} \rho_{\delta \gamma}^{\mathrm{HF}} \rho_{\eta \epsilon}^{\mathrm{HF}} .
\end{aligned}
$$

If the 3B contribution is perturbative, one would expect Eq. (44) to provide a good starting point to evaluate the full $3 \mathrm{BF}$ contribution of Eq. (41). This seems to be the case in finite nuclei where, however, the internal density matrices should be appropriately dressed [35] to find accurate results.

To close this section, let us comment on the use of effective interactions in the calculation of the ground-state energy itself. Two errors can arise in this context. The first would involve incorrectly accounting for the different pre-factor in the $1 \mathrm{~B}$ and $2 \mathrm{~B}$ effective interactions. This double counting of the HF potential was already discussed at the end of Sec. II. The second issue would arise if a 3B correction to the energy was neglected. The Hartree-Fock case provides a good example of the latter. Replacing the bare 2B interaction in Eq. (42) by the effective $2 \mathrm{~B}$ force would immediately lead to errors. The 3B correction in Eq. (42) would necessarily have to change to provide the same result. Consequently, performing a calculation with an effective $2 \mathrm{~B}$ force and simply computing the energy with the usual $2 \mathrm{~B}$ formulas is not enough. The $3 \mathrm{~B}$ correction is needed anyway and is different if one uses the bare or the effective interaction.

\section{CONCLUSIONS}

We have presented an extension of the SCGF approach to include $3 \mathrm{~B}$ interactions. The method allows one to incorporate consistently $2 \mathrm{~B}$ and $3 \mathrm{~B}$ forces on an equal footing and should be interesting for nuclear physics applications. Other manybody systems in which induced $3 \mathrm{BF}$ are generated by cuts in the model space could potentially benefit from this treatment as well.

The 3BF was introduced in two different but equivalent ways in the formalism. On the one hand, we have studied the diagrammatic perturbative expansion of the propagator including $1 \mathrm{~B}, 2 \mathrm{~B}$, and $3 \mathrm{~B}$ forces. The expansion is analogous to cases previously studied in the literature, but the $3 \mathrm{BF}$ requires some careful handling. We present in Appendix A the Feynman rules associated with this expansion. Within a SCGF approach, where propagators are dressed and the Dyson equation is used iteratively, only 1PI skeleton diagrams enter the expansion. The number of diagrams can be further reduced by introducing effective interactions, which sum up a subseries of interaction-reducible diagrams. These effective interactions can be interpreted as a generalization of the normal ordering of the many-body Hamiltonian. Instead of ordering with respect to an uncorrelated state, however, the effective interactions include the effect of many-body correlations by construction. The proper self-energy can be defined from 1B, 2B, and 3B forces and still be computed within the Dyson's equation. We have shown how this effective grouping of operators reduces the number of diagrams by considering the perturbation expansion up to third order. The equivalence between the original diagrammatic expansion and that obtained from the effective interaction at any arbitrary order is proven in Appendix B.

On the other hand, the propagator method can be expressed using the EOM. We have re-derived the basic equations of this method, consistently including 3BFs. The Martin-Schwinger hierarchy now connects the $(n)$-body propagator to the $(n-1)-$, the $(n+1)-$, and, via the 3BF, the $(n+2)$-body GF. In turn, this requires the introduction of vertex functions beyond the four-point level. Through the hierarchy of the EOM, we have found an expression for the vertex function $\Gamma^{4-p t}$, which embodies the higher order interacting contributions beyond the mean field. Truncation to second order of this function, together with complete second-order expression for the sixpoint $\Gamma$ function, provides the third-order approximation for the irreducible self-energy. The correspondence to the diagrams obtained in the perturbative expansion indicates that these two different approaches are equivalent.

Moreover, we have shown that, using the 2B effective interaction in truncation schemes based on $\Gamma^{4-p t}$, leads to either ladder, ring or parquet approximations that effectively include some 3B terms. Within these approximations, the general structure of the formalism, based on $2 \mathrm{BFs}$, remains unaltered [14]. Results obtained recently both in calculations for infinite nuclear matter [27] as well as nuclei [35] exploit this expanded self-consistent Green's functions approach to include 3B nuclear forces.

More importantly, however, this approach is able to provide a general mechanism to devise nonperturbative resummation schemes. In particular, we have proposed a potentially relevant correction to the self-energy that includes interactionirreducible three-body effects explicitly. Extensions to include $3 \mathrm{BFs}$ beyond the effective $2 \mathrm{BF}$ approach are lacking in the literature and could prove to be potentially relevant in some instances, particularly in nuclear physics.

Finally, we have presented a general method to compute the energy of the many-body ground state by means of the GMK sum rule. The sum rule still allows for the calculation of the ground-state energy from the 2(n-1)-point GFs in spite of the fact that the energy itself is an $n$-body operator. Two possible approaches have been proposed, which require the calculation of either a $2 \mathrm{~B}$ or a $3 \mathrm{~B}$ expectation value. Depending on the relative importance of the $2 \mathrm{~B}$ and the $3 \mathrm{~B}$ forces in the system, one or the other might be preferable.

Calculations performed using this extended SCGF formalism have already been presented in the literature [27,35]. This expanded approach provides a firm basis for further studies of nuclear systems from a Green's functions point of view. The formalism can be extended to finite temperature and off-equilibrium settings. More importantly, it provides a framework to compute many relevant quantities for the description of a quantum many-body system, from binding energies, to thermodynamical properties or even pairing. On the same footing, the Gorkov-Green's function formalism for finite nuclei could be improved to include 3BFs. 
We believe that this extended approach is an interesting tool to study quantum many-body systems from an $a b$ initio microscopic point of view. In principle, the framework provides a coherent description of the correlated, nonperturbative dynamics of systems with many-body interactions. The generalization to Hamiltonians including $N$-body forces can be performed along similar lines. In addition to its academic interest, advances in interaction-tunable ultracold gases might require these developments. In nuclear physics, the importance of $4 \mathrm{BFs}$, either bare or induced, could also be assessed using analogous techniques. Ultimately, the methods presented here should be a good starting point to foster new initiatives that systematically address the issue of many-body forces in quantum many-body systems.

\section{ACKNOWLEDGMENTS}

This work is supported by the Consolider Ingenio 2010 Programme CPAN CSD2007-00042, Grant No. FIS201124154 from MICINN (Spain) and Grant No. 2009SGR-1289 from Generalitat de Catalunya (Spain), and by STFC through Grants No. ST/I005528/1 and No. ST/J000051/1. A. Carbone acknowledges the support by the SUR of the ECO from Generalitat de Catalunya.

\section{APPENDIX A: FEYNMAN DIAGRAM RULES FOR TWO- AND THREE-BODY INTERACTIONS}

We present in this appendix the Feynman rules associated with the diagrams arising in the perturbative expansion of Eq. (7). The rules are given both in time and energy formulation, and some specific examples will be considered at the end. We pay particular attention to nontrivial symmetry factors arising in diagrams that include many-body interactions. We work with antisymmetrized matrix elements, but for practical purposes represent them by extended lines.

We provide the Feynman diagram rules for a given $p$-body propagator, such as Eqs. (3) and (4). These arise from a trivial generalization of the perturbative expansion of the $1 \mathrm{~B}$ propagator in Eq. (7). At $k$ th order in perturbation theory, any contribution from the time-ordered product in Eq. (7), or its generalization, is represented by a diagram with $2 p$ external lines and $k$ interaction lines (from here on called vertices), all connected by means of oriented fermion lines. These fermion lines arise from contractions between annihilation and creation operators,

$$
a_{\delta}^{I}(t) a_{\gamma}^{I \dagger}\left(t^{\prime}\right) \equiv\left\langle\Phi_{0}^{N}\left|\mathcal{T}\left[a_{\delta}^{I}(t) a_{\gamma}^{I \dagger}\left(t^{\prime}\right)\right]\right| \Phi_{0}^{N}\right\rangle=i \hbar G_{\delta \gamma}^{(0)}\left(t-t^{\prime}\right) .
$$

Applying the Wick theorem to any such arbitrary diagram, results in the following Feynman rules.

Rule 1: Draw all, topologically distinct and connected diagrams with $k$ vertices, and $p$ incoming and $p$ outgoing external lines, using directed arrows. For interaction vertices the external lines are not present.

Rule 2: Each oriented fermion line represents a Wick contraction, leading to the unperturbed propagator $i \hbar G_{\alpha \beta}^{(0)}\left(t-t^{\prime}\right)$ [or $i \hbar G_{\alpha \beta}^{(0)}\left(\omega_{i}\right)$ ]. In time formulation, the $t$ and $t^{\prime}$ label the times of the vertices at the end and at the beginning of the line. In energy formulation, $\omega_{i}$ denotes the energy carried by the propagator.

Rule 3: Each fermion line starting from and ending at the same vertex is an equal-time propagator, $-i \hbar G_{\alpha \beta}^{(0)}\left(0^{-}\right)=$ $\rho_{\alpha \beta}^{(0)}$.

Rule 4: For each 1B, 2B or 3B vertex, write down a factor $\frac{i}{\hbar} U_{\alpha \beta},-\frac{i}{\hbar} V_{\alpha \gamma, \beta \delta}$ or $-\frac{i}{\hbar} W_{\alpha \gamma \xi, \beta \delta \theta}$, respectively. For effective interactions, the factors are $-\frac{\mathrm{i}}{\hbar} \widetilde{U}_{\alpha \beta},-\frac{\mathrm{i}}{\hbar} \widetilde{V}_{\alpha \gamma, \beta \delta}$.

When propagator renormalization is considered, only skeleton diagrams are used in the expansion. In that case, the previous rules apply with the substitution $i \hbar G_{\alpha \beta}^{(0)} \rightarrow i \hbar G_{\alpha \beta}$. Furthermore, note that Rule 3 applies to diagrams embedded in the one-body effective interaction (see Fig. 1) and therefore they should not be considered explicitly in an interactionirreducible expansion. In calculating $\tilde{U}$, however, one should use the correlated $\rho_{\alpha \beta}$ instead of the unperturbed one.

Rule 5: Include a factor $(-1)^{L}$ where $L$ is the number of closed fermion loops. This sign comes from the odd permutation of operators needed to create a loop and does not include loops of a single propagator, already accounted for by Rule 3.

Rule 6: For a diagram representing a $2 p$-point GF, add a factor $(-i / \hbar)$, whereas for a $2 p$-point interaction vertex without external lines (such as $\Sigma^{\star}$ and $\Gamma^{2 p-p t}$ ) add a factor $i \hbar$.

The next two rules require a distinction between the time and the energy representation. In the time representation,

Rule 7: Assign a time to each interaction vertex. All the fermion lines connected to the same vertex $i$ share the same time $t_{i}$.

Rule 8: Sum over all the internal quantum numbers and integrate over all internal times from $-\infty$ to $+\infty$.

Alternatively, in energy representation,

Rule $7^{\prime}$ : Label each fermion line with an energy $\omega_{i}$, under the constraint that the total incoming energy equals the total outgoing energy at each interaction vertex, $\sum_{i} \omega_{i}^{\text {in }}=$ $\sum_{i} \omega_{i}^{\text {out }}$.

Rule 8': Sum over all the internal quantum numbers and integrate over each independent internal energy, with an extra factor $\frac{1}{2 \pi}$, i.e., $\int_{-\infty}^{+\infty} \frac{d \omega_{i}}{2 \pi}$.

Each diagram is then multiplied by a combinatorial factor $S$ that originates from the number of equivalent Wick contractions that lead to it. This symmetry factor represents the order of the symmetry group for one specific diagram or, in other words, the order of the permutation group of both open and closed lines, once the vertices are fixed. Its structure, assuming only $2 \mathrm{BFs}$ and $3 \mathrm{BFs}$, is the following:

$$
S=\frac{1}{k !} \frac{1}{\left[(2 !)^{2}\right]^{q}\left[(3 !)^{2}\right]^{k-q}}\left(\begin{array}{c}
k \\
q
\end{array}\right) C=\prod_{i} S_{i} .
$$




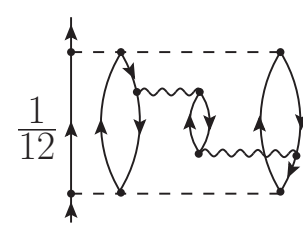

(a)

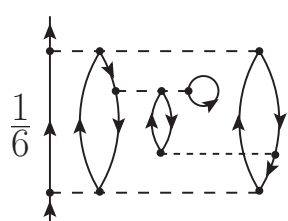

(c)

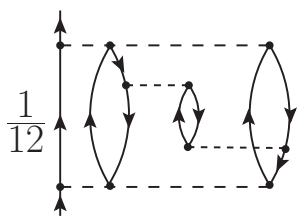

(b)

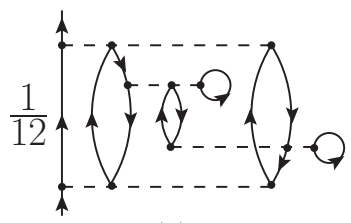

(d)
FIG. 16. Examples of diagrams containing symmetric and interacting lines, with explicit symmetry factors. Diagrams (b) to (d) are obtained by expanding the effective interaction of diagram (a) according to Eq (11). Swapping the $3 \mathrm{~B}$ and $2 \mathrm{~B}$ internal vertices in (c) gives a distinct, but topologically equivalent, contribution.

Here, $k$ represents the order of expansion. $q(k-q)$ denotes the number of $2 \mathrm{~B}(3 \mathrm{~B})$ vertices in the diagram. The binomial factor counts the number of terms in the expansion $(V+W)^{k}$ that have $q$ factors of $V$ and $k-q$ factors of $W$. Finally, $C$ is the overall number of distinct contractions and reflects the symmetries of the diagram. Stating general rules to find $C$ is not simple. For example, explicit simple rules valid for the well-known $\lambda \phi^{4}$ scalar theory are still an object of debate [68]. An explicit calculation for $C$ has to be carried out diagram by diagram [68]. Equation (A1) can normally be factorized in a product factor $S_{i}$, each due to a particular symmetry of the diagram. In the following, we list a series of specific examples which is, by all means, not exhaustive.

Rule 9: For each group of $n$ symmetric lines, or symmetric groups of lines as defined below, multiply by a symmetry factor $S_{i}=\frac{1}{n !}$. The overall symmetry factor of the diagram will be $S=\prod_{i} S_{i}$. Possible cases include the following:

(i) Equivalent lines. $n$ equally oriented fermion lines are said to be equivalent if they start from the same initial vertex and end on the same final vertex.

(ii) Symmetric and interacting lines. $n$ equally oriented fermion lines that start from the same initial vertex and end on the same final vertex, but are linked via an interaction vertex to one or more close fermion line blocks. The factor arises as long as the diagram is invariant under the permutation of the two blocks.

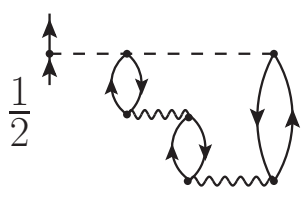

(a)

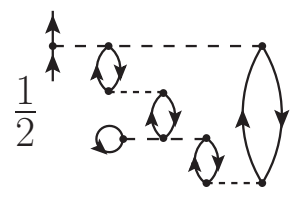

(b)

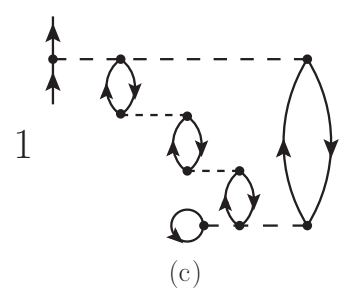

FIG. 17. Examples of diagrams entering the static part of the self-energy. Applying rule 9-ii, diagrams (a) and (b) take a factor $S_{s i}=\frac{1}{2}$ from the symmetry between the two bubbles attached to the upper three body vertex. The symmetry is broken in diagram (c), where the overall factor is $S_{s i}=1$

(iii) Equivalent groups of lines. These are blocks of interacting lines (e.g., series of bubbles) that are equal to each other: They all start from the same initial vertex and end on the same final vertex.

Rule 9-i is the most well-known case and applies, for instance, to the two second-order diagrams of Fig. 3. Diagram Fig. 3(a) has two upward-going equivalent lines and requires a symmetry factor $S_{e}=\frac{1}{2 !}$. In contrast, diagram Fig. 3(b) has three upward-going equivalent lines and two downward-going equivalent lines, that give a factor $S_{e}=\frac{1}{2 ! 3 !}=\frac{1}{12}$.

Figures 16 and 17 give specific examples of the application of rule 9-ii. Diagram Fig. 16(a) has three upward-going equivalent, noninteracting lines, which yield a symmetry factor $S_{e}=\frac{1}{3 !}$ due to rule 9-i. However, there are also two downward-going symmetric and equivalent lines, that interact through the exchange of a bubble and thus give rise to a factor $S_{s i}=\frac{1}{2 !}$. The total factor is therefore $S=S_{e} \times S_{s i}=$ $\frac{1}{12}$. Let us now expand the two $2 \mathrm{~B}$ effective interactions that are connected to the intermediate bubble according to Eq. (11). Diagram Fig. 16(a) is now seen to contain three contributions, diagrams Figs. 16(b)-16(d), with the symmetry factors shown in the figure. Note that drawing the contracted 3B vertex above or below the bubble in Fig. 16(c) leads to two topologically equivalent diagrams that must only be drawn once, i.e., diagram Fig. 16(c). However, because the diagram is no longer symmetric under the exchange of the

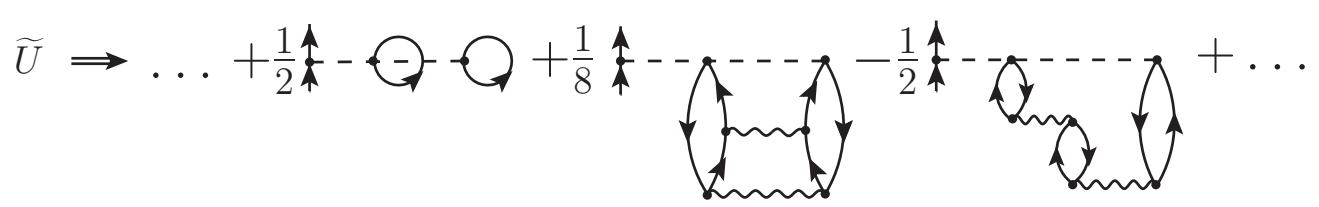

FIG. 18. Diagrams entering the effective one-body interaction, Eq. (10), obtained by substituting the right-hand side of Fig. 13 into Eq. (23). The two bubble terms correctly reproduce the symmetric factor inferred by applying rules 9-i and 9-ii. 


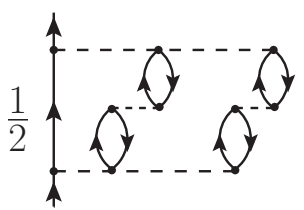

(a)

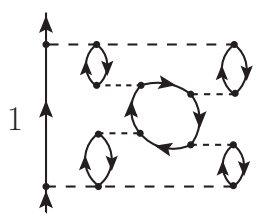

(b)
FIG. 19. Examples of a diagram where equivalent group of lines are present and one where rule 9-iii does not apply. Swapping the two chains of bubbles in (a), one finds an identical diagram. This is precisely the case of rule 9-iii, which brings in a factor $S_{e g l}=$ $\frac{1}{2}$. Performing the same exchange in diagram (b) generates a graph where the direction of the internal loop is reversed. No symmetry rule applies here and $S_{e g l}=1$

two downward-going equivalent lines, rule 9-ii does not apply anymore and the $S_{s i}$ factor is no longer needed.

A similar situation occurs when the two interacting fermion lines start and end on the same vertex, as in Fig. 17. Consider the left-most and right-most external fermion bubbles. In all three diagrams, they are connected to each other by a $3 \mathrm{~B}$ interaction vertex above and by a series of interactions and medium polarizations below. The intermediate bubble interactions in diagrams Figs. 17(a) and 17(b) are symmetric under exchange. There are therefore two sets of symmetric interacting lines (the two up-going and two down-going fermion lines) and hence both diagrams take a factor $S_{s i}=1 / 2$. In contrast, the two external loops in $17(\mathrm{c})$ are not symmetric under exchange due to the lower 3B vertex. Rule 9-ii does not apply anymore and $S_{s i}=1$. If all the vertices between the external loops were equal (e.g., effective 2B terms $\tilde{V}$ ), a factor $S_{s i}=1 / 2$ would still apply.
The case of Fig. 17 is of particular importance because these diagrams directly contribute to the energy-independent $1 \mathrm{~B}$ effective interaction. In the EOM approach, these contributions arise from the first three terms on the right-hand side of Fig. 13. Note that the ladder diagram has a symmetry factor $S_{e}=1 / 2$ and that the exchange contribution in the bubble term has to be considered. Using these diagrams to define the $2 \mathrm{~B}$ propagator in Eq. (23) and inserting these in the last term of Eq. (10), one finds the contributions to $\tilde{U}$ shown in Fig. 18. The two bubble terms have summed up to form diagram Fig. 17(a), each of them contributing a factor $1 / 4$ from Eq. (10). Consequently, the approach leads to the correct overall $S_{s i}=1 / 2$ symmetry factor. In our approach, there is no need to explicitly compute these diagrams because they are automatically included by Eq. (10).

Finally, rule 9-iii applies to the diagram in Fig. 19. In this case, the two chains of bubble diagrams are equal and start and end at the same $3 \mathrm{BF}$ vertices. Hence, they are equivalent groups of lines and the diagram takes a factor $S_{\text {egl }}=\frac{1}{2}$. Diagram Fig. 19(b) is different because the exchange of all the bubbles generates a diagram in which the direction of the internal fermion loop is reversed. Therefore no symmetry rule applies and the symmetry factor is just $S_{\text {egl }}=1$. This is, however, topologically equivalent to the initial diagram and hence must be counted only once.

As an example of the application of the above Feynman rules, we give here the formulas for some of the diagrams in Fig. 5. Let us start by a contribution that was discussed in Sec. III, diagram Fig. 5(c). There are two sets of upward-going equivalent lines, which contribute to a symmetry factor $S_{e}=$ $\frac{1}{2^{2}}$. Considering the overall factor of Eq. (A1) and the presence of one closed fermion loop, one finds

$$
\begin{aligned}
\Sigma_{\alpha \beta}^{(5 c)}(\omega)= & -\frac{(i \hbar)^{4}}{4} \int \frac{d \omega_{1}}{2 \pi} \cdots \int \frac{d \omega_{4}}{2 \pi} \sum_{\substack{\gamma \delta \nu \mu \epsilon \lambda \\
\xi \eta \theta \sigma \tau \chi}} \widetilde{V}_{\alpha \gamma, \delta \nu} G_{\delta \mu}^{(0)}\left(\omega_{1}\right) G_{\nu \epsilon}^{(0)}\left(\omega_{2}\right) W_{\mu \epsilon \lambda, \xi \eta \theta} G_{\xi \sigma}^{(0)}\left(\omega_{3}\right) G_{\eta \tau}^{(0)}\left(\omega_{4}\right) \\
& \times G_{\theta \gamma}^{(0)}\left(\omega_{1}+\omega_{2}-\omega\right) \widetilde{V}_{\sigma \tau, \beta \chi} G_{\chi \lambda}^{(0)}\left(\omega_{3}+\omega_{4}-\omega\right) .
\end{aligned}
$$

Diagrams Figs. 5(h) and 5(i) differ only for the orientation of a loop. Hence, there are two pairs of equivalent lines in the first case and one pair and one triplet of equivalent lines in the second, which is reflected in their different symmetry factors:

$$
\begin{aligned}
\Sigma_{\alpha \beta}^{(5 h)}(\omega)= & \frac{(i \hbar)^{5}}{4} \int \frac{d \omega_{1}}{2 \pi} \cdots \int \frac{d \omega_{5}}{2 \pi} \sum_{\substack{\gamma \delta \epsilon \epsilon \\
\xi \theta \sigma \mu \nu \lambda \\
\eta \tau \phi \chi \zeta}} \widetilde{V}_{\alpha \gamma, \delta \epsilon} G_{\delta \xi}^{(0)}\left(\omega_{1}\right) G_{\nu \gamma}^{(0)}\left(\omega_{2}\right) W_{\xi \theta \sigma, \mu \nu \lambda} G_{\mu \eta}^{(0)}\left(\omega_{3}\right) G_{\chi \theta}^{(0)}\left(\omega_{4}\right) \\
& \times G_{\epsilon \tau}^{(0)}\left(\omega-\omega_{1}+\omega_{2}\right) W_{\eta \tau \phi, \beta \chi \zeta} G_{\lambda \phi}^{(0)}\left(\omega_{5}\right) G_{\zeta \sigma}^{(0)}\left(\omega_{2}+\omega_{3}+\omega_{5}-\omega_{1}-\omega_{4}\right), \\
\Sigma_{\alpha \beta}^{(5 i)}(\omega)= & \frac{(i \hbar)^{5}}{12} \int \frac{d \omega_{1}}{2 \pi} \cdots \int \frac{d \omega_{5}}{2 \pi} \sum_{\substack{\xi \delta \epsilon \\
\xi \theta \sigma \mu \nu \lambda \\
\eta \tau \phi \chi \zeta}} \widetilde{V}_{\alpha \gamma, \delta \epsilon} G_{\delta \xi}^{(0)}\left(\omega_{1}\right) G_{\epsilon \theta}^{(0)}\left(\omega_{2}\right) W_{\xi \theta \sigma, \mu \nu \lambda} G_{\mu \eta}^{(0)}\left(\omega_{3}\right) G_{\nu \tau}^{(0)}\left(\omega_{4}\right) \\
& \times G_{\chi \gamma}^{(0)}\left(\omega_{1}+\omega_{2}-\omega\right) W_{\eta \tau \phi, \beta \chi \zeta} G_{\lambda \phi}^{(0)}\left(\omega_{5}\right) G_{\zeta \sigma}^{(0)}\left(\omega_{3}+\omega_{4}+\omega_{5}-\omega_{1}-\omega_{2}\right) .
\end{aligned}
$$




\section{APPENDIX B: INTERACTION-IRREDUCIBLE DIAGRAMS WITH EFFECTIVE 1B AND 2B INTERACTIONS AT ALL ORDERS}

Interaction-irreducible diagrams can be used to distinguish between two different many-body effects in the SCGF approach. On the one hand, effective interactions sum all the instantaneous contributions associated with "averaging out" subgroups of particles that lead to interaction-reducible diagrams. This has the advantage of reducing drastically the number of diagrams at each order in the perturbative expansion. It also gives rise to well-defined in-medium interactions. On the other hand, the remaining diagrams will now include higher-order terms summed via the effective interaction itself.
In this appendix, we prove that the perturbative expansion can be recast into a set containing only interaction-irreducible diagrams at any given order, as long as properly defined effective interactions are used. The argument we propose was often used to demonstrate how disconnected diagrams cancel out in the perturbative expansion. We now apply it to a slightly different case that requires extra care. We focus on the case of a diagram that includes only $2 \mathrm{~B}$ and $3 \mathrm{BF}$. The extension to the general case of many-body forces should be straightforward.

Equation (7) gives the perturbative expansion of the $1 \mathrm{~B} \mathrm{GF}$ in terms of the Hamiltonian, $H(t)$, in the interaction picture. The $k$ th order term of the perturbative expansion reads

$$
G_{\alpha \beta}^{(k-\text { th })}\left(t-t^{\prime}\right)=\left(\frac{-i}{\hbar}\right)^{k+1} \frac{1}{k !} \underbrace{\int \cdots \int d t_{k}}_{k \text { terms }}\left\langle\Phi_{0}^{N}\left|\mathcal{T}\left[a_{\alpha}^{I}(t) a_{\beta}^{I \dagger}\left(t^{\prime}\right) H\left(t_{1}\right) \cdots H\left(t_{k}\right)\right]\right| \Phi_{0}^{N}\right\rangle_{\text {conn }}
$$

Only connected contributions are allowed and we take the interaction picture external creation and destruction operators to the left for convenience. Let us assume, without loss of generality, that the diagram is composed of $q 2 \mathrm{~B}$ and $k-q$ 3B interaction operators. This gives rise to $\left(\begin{array}{l}k \\ q\end{array}\right)$ identical contributions when expanding $H(t)=T(t)+V(t)+W(t)$ in the time-ordered product, as discussed right after Eq. (A1) above.

Let us denote with $O(t)$ a generic operator, representing either a $2 \mathrm{~B}, V(t)$, or a $3 \mathrm{~B}, W(t)$, potential. Suppose now that there is a subset of $m$ operators that are arbitrary connected to each other, but that share the external links with a unique operator, $O\left(t_{n}\right)$, outside the subset. In other words, $O\left(t_{n}\right)$ is the only way to enter and exit the subset of $m$ operators $\left\{O\left(t_{n+1}\right), \cdots, O\left(t_{n+m}\right)\right\}$ as drawn below:

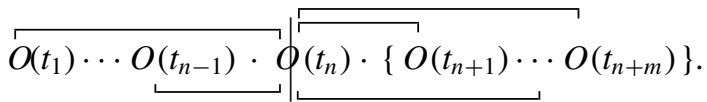

$O\left(t_{n}\right)$ is also necessarily connected to the other interactions and, hence, this is an articulation vertex. In general, there can be an arbitrary number of articulation vertices, such as $O\left(t_{n}\right)$, at any given order. Each one of these vertices would isolate a particular subset of operators. The following arguments can be applied to each subset separately.

For simplicity, let us restrict the argument to the simplest case of one articulation vertex only. Suppose that, among $m$ terms, there are $a 2 \mathrm{~B}$ and $b 3 \mathrm{~B}$ interactions, with $a+b=m$. The number of time-ordered products $V(t)$ and $W(t)$ in Eq. (B1) that is consistent with the above decomposition is

$$
\left(\begin{array}{l}
k \\
q
\end{array}\right)\left(\begin{array}{l}
q \\
a
\end{array}\right)\left(\begin{array}{c}
k-q \\
b
\end{array}\right)=\left(\begin{array}{l}
k \\
m
\end{array}\right)\left(\begin{array}{l}
m \\
a
\end{array}\right)\left(\begin{array}{c}
n \\
q-a
\end{array}\right),
$$

where $m+n=k$.

Let us consider the case in which $O\left(t_{n}\right)$ is a $3 \mathrm{~B}$ operator, with matrix elements $W_{\mu \gamma \delta, \theta \sigma \xi}$ connected with four legs to the internal subset of $m$ vertices and with two legs to the rest of the diagram. We can factorize the amplitude in Eq. (B1) by adding an intermediate identity operator as follows:

$$
\begin{aligned}
& \frac{1}{n !}\left(\begin{array}{c}
n \\
q-a
\end{array}\right) \underbrace{\int \cdots \int_{n} d t_{n}}_{n \text { terms }}\left\langle\Phi_{0}^{N}\left|\mathcal{T}\left[a_{\alpha}^{I}(t) a_{\beta}^{I \dagger}\left(t^{\prime}\right) O\left(t_{1}\right) \cdots O\left(t_{n-1}\right) a_{\mu}^{I \dagger}\left(t_{n}^{+}\right) a_{\theta}^{I}\left(t_{n}\right)\right]\right| \Phi_{0}^{N}\right\rangle W_{\mu \gamma \delta, \theta \sigma \xi} \frac{1}{(3 !)^{2}}\left(\begin{array}{c}
3 \\
1
\end{array}\right)^{2} \\
& \quad \times \frac{1}{(m) !}\left(\begin{array}{c}
m \\
a
\end{array}\right) \underbrace{\int \cdots \int_{k} d t_{k}}_{m \text { terms }}\left\langle\Phi_{0}^{N}\left|\mathcal{T}\left[a_{\gamma}^{I \dagger}\left(t_{n}^{+}\right) a_{\delta}^{I \dagger}\left(t_{n}^{+}\right) a_{\xi}^{I}\left(t_{n}\right) a_{\sigma}^{I}\left(t_{n}\right) O\left(t_{n+1}\right) \cdots O\left(t_{k}\right)\right]\right| \Phi_{0}^{N}\right\rangle \delta_{k, n+m}
\end{aligned}
$$

Note that the factorization of the time-ordered product, by inserting a $\left|\Phi_{0}^{N}\right\rangle\left\langle\Phi_{0}^{N}\right|$, is possible because the Wick theorem normal-orders these products with respect to the reference state, $\left|\Phi_{0}^{N}\right\rangle$. In other words, both Eqs. (B1) and (B4) lead to exactly the same results after all Wick contractions have been carried out.

All possible orders in which a general $O(t)$ enters Eq. (B4) are equivalent and are accounted for by the binomial factors.
The factor $\left(\begin{array}{l}3 \\ 1\end{array}\right)$ accounts for all the possible ways, eventually decided by contractions, in which the six creation/annihilation operators in $W\left(t_{n}\right)$ can be separated in the two factors [see also Eq. (B7) below]. We also include an additional factor $\left(\begin{array}{l}3 \\ 1\end{array}\right)$ coming from all the possible ways to choose one creation/annihilation operator among the three possible pairs. The correct time ordering for creation and annihilation operators associated with $W\left(t_{n}\right)$ is preserved using $a^{\dagger}\left(t_{n}^{+}\right)$. 
With this decomposition, we can identify the second line of Eq. (B4) as an $m$ th order contribution (with $a \quad 2 \mathrm{~B}$ and $m-a$ 3B operators) to the perturbative expansion of $G_{\sigma \xi, \gamma \delta}^{4-\mathrm{pt}}\left(t_{n}, t_{n} ; t_{n}^{+}, t_{n}^{+}\right)=G_{\sigma \xi, \gamma \delta}^{I I}\left(t_{n}-t_{n}^{+}\right)$.
Collecting all possible contributions of form (B2) and (B4) in which the first $n$ operators are unchanged, the $k$ th order interaction-reducible contribution to $G$ becomes

$$
\begin{gathered}
G_{\alpha \beta}^{(k-\text { th) }}\left(t-t^{\prime}\right) \rightarrow\left(\frac{-i}{\hbar}\right)^{n+1} \frac{1}{n !}\left(\begin{array}{c}
n \\
q-a
\end{array}\right) \int \cdots \int d t_{n}\left\langle\Phi_{0}^{N}\left|\mathcal{T}\left[a_{\alpha}^{I}(t) a_{\beta}^{I^{\dagger}}\left(t^{\prime}\right) O\left(t_{1}\right) \cdots O\left(t_{n-1}\right) a_{\mu}^{I \dagger}\left(t_{n}^{+}\right) a_{\theta}^{I}\left(t_{n}\right)\right]\right| \Phi_{0}^{N}\right\rangle_{\text {int-irr }} \\
\times \frac{W_{\mu \gamma \delta, \theta \sigma \xi} \frac{i \hbar}{(2 !)^{2}} G_{\sigma \xi, \gamma \delta}^{I I(m-\text { th, a) }}\left(t_{n}-t_{n}^{+}\right)}{U_{\mu \theta}^{\text {eff }}}
\end{gathered}
$$

where $G^{I I(m-\text { th,a) }}$ sums all the diagrams at $m$ th order with $a$ two-body operators. Note that the last term no longer depends on time and can be seen as an energy-independent correction to the $1 \mathrm{~B}$ potential. We can automatically take into account these interaction-reducible terms by reformulating the initial Hamiltonian to include the effective 1B vertex:

$$
\widetilde{U}_{\mu \theta} \rightarrow U_{\mu \theta}+W_{\mu \gamma \delta, \theta \sigma \xi} \frac{i \hbar}{(2 !)^{2}} \frac{G_{\sigma \xi, \gamma \delta}^{I I}\left(t-t^{+}\right),}{-\frac{\dot{i}}{\hbar} \rho_{\sigma \xi, \gamma \delta}^{2 B}}
$$

where now we use an exact $G^{I I}$. The perturbative expansion obtained with this effective interaction should only contain interaction-irreducible diagrams to avoid double counting.

Note that in Eq. (B5) we automatically obtain the correct symmetry factor $1 /(2 !)^{2}$ associated with the contraction of $W$ with the two pairs of incoming and outgoing lines of $G^{I I}$. In the general case, a $c$-body vertex can be reduced to a $d$-body one (with $d<c$ ) by using a $(c-d)$-body GF. The overall combinatorial factor in that case will be

$$
\frac{1}{(c !)^{2}}\left(\frac{c !}{d !(c-d) !}\right)^{2}=\underbrace{\frac{1}{(d !)^{2}}}_{\text {new vertex }} \underbrace{\frac{1}{((c-d) !)^{2}}}_{c-d \text { equal lines }} .
$$

This yields both the correct combinatorial factors entering the new effective $d$-body vertex and the symmetry factor associated with the contraction with the $(c-d)$-body GF. The above arguments can be generalized to any starting $n$-body Hamiltonian. Applying these derivations to all possible cases for a 3B Hamiltonian leads to the effective interactions discussed in Eqs. (10) and (11).
[1] P. Ring and P. Schuck, The Nuclear Many-Body Problem, 1st ed. (Springer, New York, 1980).

[2] A. L. Fetter and J. D. Walecka, Quantum Theory of ManyParticle System, 1st ed. (McGraw-Hill, New York, 1971).

[3] W. H. Dickhoff and D. V. Neck, Many-Body Theory Exposed!, 2nd ed. (World Scientific Publishing, London, 2005).

[4] R. D. Mattuck, A Guide to Feynman Diagrams in the Many-Body Problem (Dover Publications, New York, 1992).

[5] P. C. Martin and J. Schwinger, Phys. Rev. 115, 1342 (1959).

[6] A. A. Abrikosov, L. P. Gorkov, and I. E. Dzyaloshinski, Methods of Quantum Field Theory in Statistical Physics (Dover, New York, 1975).

[7] F. Aryasetiawan and O. Gunnarsson, Rep. Prog. Phys. 61, 237 (1998).

[8] G. Onida, L. Reining, and A. Rubio, Rev. Mod. Phys. 74, 601 (2002).

[9] W. von Niessen, J. Schirmer, and L. Cederbaum, Comp. Phys. Rep. 1, 57 (1984).

[10] J. V. Ortiz, WIREs Comput. Mol. Sci. 3, 123 (2013).

[11] C. Barbieri, D. Van Neck, and W. H. Dickhoff, Phys. Rev. A 76, 052503 (2007).

[12] M. Degroote, D. Van Neck, and C. Barbieri, Phys. Rev. A 83, 042517 (2011).

[13] H. Müther and A. Polls, Prog. Part. Nucl. Phys. 45, 243 (2000).

[14] W. H. Dickhoff and C. Barbieri, Prog. Part. Nucl. Phys. 52, 377 (2004).

[15] C. Barbieri and W. H. Dickhoff, Phys. Rev. C 65, 064313 (2002).
[16] C. Barbieri and M. Hjorth-Jensen, Phys. Rev. C 79, 064313 (2009).

[17] V. Somà, C. Barbieri, and T. Duguet, Phys. Rev. C 87, 011303(R) (2013).

[18] A. Ramos, A. Polls, and W. H. Dickhoff, Nucl. Phys. A 503, 1 (1989)

[19] T. Frick and H. Müther, Phys. Rev. C 68, 034310 (2003).

[20] A. Rios, A. Polls, and I. Vidaña, Phys. Rev. C 79, 025802 (2009).

[21] V. Somà and P. Bożek, Phys. Rev. C 78, 054003 (2008).

[22] P. Grangé, A. Lejeune, M. Martzolff, and J.-F. Mathiot, Phys. Rev. C 40, 1040 (1989).

[23] W. Zuo, A. Lejeune, U. Lombardo, and J. Mathiot, Nucl. Phys. A 706, 418 (2002).

[24] Z. H. Li, U. Lombardo, H.-J. Schulze, W. Zuo, L. W. Chen, and H. R. Ma, Phys. Rev. C 74, 047304 (2006).

[25] K. Hebeler, S. K. Bogner, R. J. Furnstahl, A. Nogga, and A. Schwenk, Phys. Rev. C 83, 031301(R) (2011).

[26] A. Lovato, O. Benhar, S. Fantoni, and K. E. Schmidt, Phys. Rev. C 85, 024003 (2012).

[27] A. Carbone, A. Polls, and A. Rios, Phys. Rev. C 88, 044302 (2013).

[28] S. C. Pieper and R. B. Wiringa, Ann. Rev. Nucl. Part. Sci. 51, 53 (2001).

[29] R. B. Wiringa and S. C. Pieper, Phys. Rev. Lett. 89, 182501 (2002).

[30] P. Navrátil, V. G. Gueorguiev, J. P. Vary, W. E. Ormand, and A. Nogga, Phys. Rev. Lett. 99, 042501 (2007). 
[31] G. Hagen, T. Papenbrock, D. J. Dean, A. Schwenk, A. Nogga, M. Włoch, and P. Piecuch, Phys. Rev. C 76, 034302 (2007).

[32] T. Otsuka, T. Suzuki, J. D. Holt, A. Schwenk, and Y. Akaishi, Phys. Rev. Lett. 105, 032501 (2010).

[33] R. Roth, S. Binder, K. Vobig, A. Calci, J. Langhammer, and P. Navrátil, Phys. Rev. Lett. 109, 052501 (2012).

[34] G. Hagen, M. Hjorth-Jensen, G. R. Jansen, R. Machleidt, and T. Papenbrock, Phys. Rev. Lett. 108, 242501 (2012).

[35] A. Cipollone, C. Barbieri, and P. Navrátil, Phys. Rev. Lett. 111, 062501 (2013).

[36] K. Morawetz, Phys. Rev. C 63, 014609 (2000).

[37] K. Hebeler and A. Schwenk, Phys. Rev. C 82, 014314 (2010).

[38] S. Binder, P. Piecuch, A. Calci, J. Langhammer, P. Navrátil, and R. Roth, arXiv:1309.1123 [nucl-th].

[39] V. M. Galitskii and A. B. Migdal, Sov. Phys. JETP 7, 96 (1958).

[40] D. S. Koltun, Phys. Rev. 9, 484 (1974).

[41] R. Rajaraman and H. Bethe, Rev. Mod. Phys. 39, 745 (1967).

[42] V. G. J. Stoks, R. A. M. Klomp, C. P. F. Terheggen, and J. J. de Swart, Phys. Rev. C 49, 2950 (1994).

[43] R. B. Wiringa, V. G. J. Stoks, and R. Schiavilla, Phys. Rev. C 51, 38 (1995).

[44] R. Machleidt, Phys. Rev. C 63, 024001 (2001).

[45] S. C. Pieper, V. R. Pandharipande, R. B. Wiringa, and J. Carlson, Phys. Rev. C 64, 014001 (2001).

[46] E. Epelbaum, H.-W. Hammer, and U.-G. Meisner, Rev. Mod. Phys. 81, 1773 (2009).

[47] R. Machleidt and D. R. Entem, Phys. Rep. 503, 1 (2011).

[48] S. K. Bogner, R. J. Furnstahl, and A. Schwenk, Prog. Part. Nucl. Phys. 65, 94 (2010).
[49] P. R. Johnson, E. Tiesinga, J. V. Porto, and C. J. Williams, New J. Phys. 11, 093022 (2009).

[50] A. Kievsky, E. Garrido, C. Romero-Redondo, and P. Barletta, Few-Body Syst. 51, 259 (2011).

[51] J. P. Blaizot and G. Ripka, Quantum Theory of Finite Systems, 1st ed. (MIT Press, Cambridge, 1986).

[52] F. Capuzzi and C. Mahaux, Ann. Phys. 245, 147 (1996).

[53] L. S. Cederbaum, Ann. Phys. 291, 169 (2001).

[54] C. Barbieri and B. K. Jennings, Phys. Rev. C 72, 014613 (2005).

[55] R. J. Charity, L. G. Sobotka, and W. H. Dickhoff, Phys. Rev. Lett. 97, 162503 (2006).

[56] G. Baym and L. P. Kadanoff, Phys. Rev. 124, 287 (1961).

[57] G. Baym, Phys. Rev. 127, 1391 (1962).

[58] V. Somà and P. Bożek, Phys. Rev. C 80, 025803 (2009).

[59] Z. H. Li and H.-J. Schulze, Phys. Rev. C 85, 064002 (2012).

[60] R. Mattuck and A. Theumann, Adv. Phys. 20, 721 (1971).

[61] J. Schirmer, L. S. Cederbaum, and O. Walter, Phys. Rev. A 28 , 1237 (1983).

[62] D. Danovich, WIREs Comput. Mol. Sci. 1, 377 (2011).

[63] E. Bergli and M. Hjorth-Jensen, Ann. Phys. 326, 1125 (2011).

[64] A. Rios, A. Polls, A. Ramos, and H. Müther, Phys. Rev. C 74, 054317 (2006).

[65] T. Duguet and G. Hagen, Phys. Rev. C 85, 034330 (2012).

[66] M. Bender and P.-H. Heenen, Rev. Mod. Phys. 75, 121 (2003).

[67] A. Günther, R. Roth, H. Hergert, and S. Reinhardt, Phys. Rev. C 82, 024319 (2010); E. N. E. van Dalen, P. Gögelein, and H. Müther, ibid. 80, 044312 (2009).

[68] L. Hue, H. Hung, and H. Long, Rep. Math. Phys. 69, 331 (2012). 